\title{
The role of particle surface functionality and microstructure development in isothermal and non-isothermal crystallization behavior of Polyamide 6/Cellulose nanocrystals nanocomposites
}

\author{
Shahab Kashani Rahimi and Joshua U Otaigbe* \\ School of Polymers and High Performance Materials, The University of Southern Mississippi, 118 College \\ Drive, Hattiesburg, MS, USA \\ * Corresponding Author: Email address: Joshua.otaigbe@usm.edu \\ Phone: (601) 266-5596, Fax: (601) 266-5504
}

Revised and Submitted to $\underline{\text { Polymer }}$, October 28, 2016 


\begin{abstract}
:
Polyamide 6 (PA6)/cellulose nanocrystal (CNC) and aminopropyl triethoxy silane (APS) modified CNC nanocomposites were prepared by in situ anionic ring opening polymerization and subsequent melt extrusion. The morphological observation of these hybrid systems revealed that the non-modified nanocrystals developed a network-like fibrillar structure while the APS-modified CNCs were finely dispersed mostly as individual whiskers. The isothermal and non-isothermal crystallization kinetics was extensively studied with emphasis on the effects of CNC surface functionality and the subsequent microstructure development on crystallization behavior of these novel nanocomposite systems. The non-modified CNC particles with corresponding fibrillar microstructure were found significantly hinder the crystallization process and spherultic growth of polyamide 6 chains under both isothermal and non-isothermal conditions. On other hand, the surface modified cellulose nanocrystals with improved sub-micron dispersion enhance crystal nucleation in early stages of crystallization while imposing opposite effect in later stages of crystallization resulting in development of relatively smaller defective spherulitic structures.
\end{abstract}

Keywords: Crystallization kinetics, cellulose nanocrystals, cellulose whiskers, Polyamide 6 nanocomposite, Crystallization activation energy 


\section{Introduction}

Polyamide 6 (PA6) is one of the most important semi-crystalline engineering thermoplastic materials that has been of great interest both in academic studies and industrial applications due to its superior mechanical strength, chemical resistance towards wide range of solvents and hydrocarbons [1,2] and high stiffness. It is well known that there are two crystalline structures in PA6, the $\alpha$-type and $\gamma$-type polymorphs [3-5]. The $\alpha$-form is the most thermodynamically stable crystalline form of PA6 consisting of anti-parallel chains connected with the adjacent chains through hydrogen bonds in a planar zig-zag conformation while the $\gamma$-form is thermodynamically less stable consisting of pleated sheets of hydrogen bonded parallel chains $[6,7]$.

In the past decade, application of nanomaterials in development of polymer nanocomposites has received much attention in the literature as an effective approach towards controlling the physical and structural properties of the host polymers like their crystallization behavior and properties. These nanomaterial inclusions include layered silicates $[8,9]$, carbon nanotubes $[10$, 11], graphene [12] and silica nanoparticles [13].

Wu et al [14] studied the effect of montmorillonite and saponite nanoparticles on the crystallization behavior of PA6. Their results indicated that the addition of these clay nanoparticles acted as effective heterogeneous nucleating agents in the PA6 matrix at lower clay contents indicated by lower crystallization activation energy. In a reported study by Paul et al [15], it was shown that organically modified clay (O-MMT) significantly reduced the crystallization half time $\left(t_{1 / 2}\right)$ of PA6 matrix during non-isothermal crystallization followed by a complicated crystallization mechanism.

Guo and co-workers [16] studied the effect of halloysite nanotubes on non-isothermal crystallization behavior of PA6. Their results revealed that the nanotubes acted as nucleating agents in the PA6 melt while severely restricting the polymer chain mobility. It was also observed that development of $\gamma$-phase crystals were significantly promoted in the presence of halloysite nanotubes.

In a series of studies by $\mathrm{Li}$ and co-workers [17, 18], the effect of carbon nanotubes on isothermal and non-isothermal crystallization behavior of PA6 was investigated. It was found that the CNTs accelerated the nucleation phase as indicated by a shift of onset crystallization temperature towards higher temperatures as well as increased the crystallization half-time. 
However, it is worthy to note that during the isothermal crystallization, the authors reported a shortened crystallization half-time that points to fast crystallization rate, suggesting special effects of the nanomaterials in the crystallization process under the various conditions studied.

In addition, cellulose based nanomaterials in the form of nanofibers and nanocrystals, as in the current study described in this article, have also been the subject of intense study over the past years for application in polymer composites and nanocomposites due to a number of special advantages of the cellulosic nanomaterials over traditional synthetic fibers and fillers. These advantages include such as having lower density, exceptional mechanical properties, biorenewability, low cost, and potential environmentally-friendly alternative to conventional fillers $[19,20]$. In a study [21] on polylactic acid (PLA) composites reinforced with up to $10 \mathrm{wt} \%$ cellulose whiskers, it was found that the crystallization of PLA is significantly hindered and the overall crystallization of the matrix decreased. Han and co-workers [22] studied the role of cellulose whiskers (CWs) in polyurethane matrix during isothermal crystallization by using Avrami model and found that the CWs act as nucleating agents during isothermal crystallization. This effect was validated by lower activation energies of crystallization as well as shorter halftimes of the nanocomposite samples compared to that of the neat polyurethane matrix. Siqueira et al [23], in a comprehensive reported study of the effect of the shape of the cellulose nanoparticles in the form of micro-fibrillated cellulose (MFC) and CWs on the crystallization behavior of poly ( $\varepsilon$-caprolactone), showed that both MFCs and CWs can accelerate the crystallization kinetics by acting as nucleating agents under isothermal crystallization condition. In addition, the lamellar growth rate analysis showed that the MFC, and to a lesser extent, the CWs restricted the chain mobility and the lateral growth rate of the spherulites. On the other hand, other authors reported increase in relative crystallinity with addition of nanocellulose. For example, recent reported studies on polyvinyl alcohol reinforced with MFCs either cast from solution [24] or in the form of spun fibers [25] showed an increase in the degree of crystallinity in the composite samples compared to that of the neat matrix.

By contrast, studies on the properties of high melting point engineering thermoplastics such as polyamide 6 reinforced with nanocellulose has been limited due to the thermal degradation of cellulose at typical temperatures of direct melt mixing process that reduces the effectiveness and functionality of cellulose in the matrix. A recent study by Kiziltas et al [26] showed that incorporation of microcrystalline cellulose (MCC) into polyamide 6 matrix (processed with the aid of lubricant) reduced the overall crystallinity of the matrix upon addition of 2.5-30 wt\% of MCCs. 
The authors just mentioned also reported that the onset and peak crystallization temperature of the PA6 was shifted towards relatively larger values. However, the analysis of the non-isothermal crystallization of these systems via Avrami and Tobin models, showed no significant changes suggesting a poor nucleation activity of the MCC in the PA6 matrix.

In a previous study, we adopted a novel approach of in-situ Ring-Opening polymerization technique to incorporate cellulose nanocrystals (CNCs) in a PA6 matrix that gave PA6/CNC nanocomposites with improved properties such as superior physical and melt property, enhancement obtained even at very low concentration of CNCs reported in our previous publication [27]. This strategy enabled the CNCs to be incorporated in the PA6 matrix prior to processing at temperatures far below the melt processing temperature of the PA6, significantly minimizing the $\mathrm{CNC}$ thermal degradation and improving the $\mathrm{CNC}$ dispersion in the PA6 in a subsequent melt processing step. The current study described in this article reports a detailed analysis of the crystallization behavior and kinetics under both isothermal and non-isothermal conditions with specific focus on the effects of $\mathrm{CNC}$ surface modification and microstructure evolution in the PA6 matrix, and the results interpreted according to reported polymer crystallization models in the literature.

\section{Experimental}

\subsection{Materials}

The caprolactam monomer used in this study is AP-Nylon ${ }^{\circledR}$ caprolactam grade (purchased from Brueggemann Chemical, Pennsylvannia) with low moisture content (<100 ppm) suitable for anionic ring-opening polymerization. C20, a difunctional hexamehtylene-1,6- dicarbamoyl caprolactam (the ROP activator), was supplied by Brueggemann Chemical. Ethylmagnesium bromide used as the initiator was purchased from Aldrich. Aminopropyl triethoxy silane (APS) was purchased from Gelest Inc.

\subsection{Sample preparation}

The cellulose nanocrystals were prepared by sulfuric acid hydrolysis of bleached cellulose paper (obtained from Weyerhaeuser) following the method reported by Capadona et al [28] with some modifications. The cellulose paper was cut into small pieces and blended with de-ionized 
water (20 g/L) to achieve a "lumpy" cellulose pulp followed by placement in a glass reactor in an ice bath. Concentrated sulfuric acid (98\%) was slowly added to the cold suspension at $35 \mathrm{v} / \mathrm{v} \%$ of acid in the total suspension. The mixture was then heated to $50^{\circ} \mathrm{C}$ and stirred for $3.5 \mathrm{hr}$. The obtained suspension was subsequently quenched with a 10-fold excess of de-ionized (DI) water and filtered over fine fritted glass filter and washed slowly with DI water until the running water showed a $\mathrm{pH}$ of $\sim 5-6$. The resulting mixture was decanted to remove the unhydrolyzed fibers and then centrifuged ( 5 cycles) at $3000 \mathrm{rpm}$ for 10 minutes to concentrate the CNCs. The nanocrystals were recovered by freeze-drying of the suspension and were neutralized with $2 \mathrm{mM} \mathrm{NaOH}$ solution to convert the surface sulfonic acid groups to sodium salt (indicated by $\mathrm{pH}$ neutrality of the CNC dispersions). The obtained neutralized CNCs were dried overnight to constant weight in a vacuum oven at $90^{\circ} \mathrm{C}$ prior to preparation of the nanocomposite samples.

Surface modification of CNCs was carried out using amino propyl triethoxy silane (or APS). Briefly, a suspension of CNC was prepared in DI water with first mixing the CNC followed by sonication for 20 minutes. In a separate flask, the APS was added ( $7 \mathrm{wt} \%$ of the CNC) to DI water and mixed for 10 minutes in order to hydrolyze the ethoxy groups into hydroxyls. These separate mixtures were mixed and the final dispersion was allowed to mix for 3 hours under continuous agitation. The dispersion was then frozen in liquid nitrogen and freeze dried for at least 60 hours to recover the CNC powder with APS adsorbed on the surface. Finally, the polycondensation of APS with the CNC surface hydroxyl groups was carried out in vacuum oven at $120^{\circ} \mathrm{C}$ under vacuum for 1 hour.

The PA6/CNC nanocomposites were prepared by in situ ring-opening polymerization of caprolactam monomer in the presence of CNCs. For this purpose, pristine and surface modified CNCs were dispersed in the monomer at 1, 2 and $3 \mathrm{wt} \% \mathrm{CNC}$ concentrations by high-speed agitation and sonication for 25 minutes at $90^{\circ} \mathrm{C}$. This mixture was blended with another masterbatch of the activated monomer containing the initiator and activator and the resulting dispersion was infused and polymerized at $150^{\circ} \mathrm{C}$ for 30 minutes in a disk shaped mold under vacuum. The nanocomposites are named here as $\mathrm{x} \% \mathrm{CNC}$ where ' $\mathrm{x}$ ' denotes the $\mathrm{CNC}$ content in weight percent. The prepared PA6/CNC nanocomposite plates were cut and granulated into small pieces that were dried in a vacuum oven. Subsequently, the nanocomposite granules were melt processed in a second stage at $230^{\circ} \mathrm{C}$ in a Thermo Haake ${ }^{\circledR}$ minilab micro-compounder (co-rotating conical screws) in the recycle mode for 200 seconds at $120 \mathrm{rpm}$ under inert atmosphere to minimize the thermo-oxidative degradation of $\mathrm{CNC}$. 


\subsection{Microscopy}

Scanning Electron Microscopy images were acquired using a Sigma field-emission scanning electron microscope (ZEISS ${ }^{\oplus}$, USA) using a $5 \mathrm{kV}$ accelerating voltage. Images were taken from the smooth surface of the cry-fractured samples coated with silver for enhanced conductivity. Polarized optical micrographs were taken using a Nikon Optihot $2^{\oplus}$ optical microscope equipped with a Metler Toledo ${ }^{\circledR}$ hot stage. The cooling rate used for observation of isothermal crystallization from the melt phase was the maximum rate (i.e., $60^{\circ} \mathrm{C} / \mathrm{min}$ ) accessible by the instrument used. A blue filter was used for enhanced optical clarity. For POM images taken at nucleation phase, microtomed samples were used while for observation of the fully grown crystals, thin films of about $70-80 \mu \mathrm{m}$ was prepared by compression molding. Samples were heated to $250^{\circ} \mathrm{C}$ to clear the thermal history, cooled to crystallization temperature of $200^{\circ} \mathrm{C}$ followed by isothermal for 45 minutes and cooling to room temperature with the same cooling rate.

\subsection{Thermal characterization}

Crystallization and melting of the obtained PA6/CNC nanocomposites were studied using a Perkin Elmer Pyris Diamond DSC under inert nitrogen atmosphere. All the samples used were $7.5 \pm 0.2 \mathrm{mg}$ (pre-dried overnight to constant weight in vacuum oven prior to measurements) cut into a disk shape and placed in the aluminum pans to minimize the effect of uneven thermal conduction. Special care was taken to obtain similar geometry and thickness for all the tested samples. In order to measure the melting point and crystallinity, the samples were heated at $10^{\circ} \mathrm{C} / \mathrm{min}$ up to $240^{\circ} \mathrm{C}$ followed by cooling to $25^{\circ} \mathrm{C}$ and re-heated at $10^{\circ} \mathrm{C} / \mathrm{min}$ to $230^{\circ} \mathrm{C}$ so that the first and second heat runs could be obtained. In the isothermal crystallization studies, the samples were first heated up to $240^{\circ} \mathrm{C}$ at $10^{\circ} \mathrm{C} / \mathrm{min}$ and held for 4 minutes at this temperature in order to erase all previous thermal history followed by rapid quenching at $150^{\circ} \mathrm{C} /$ min to crystallization temperatures of $194^{\circ}, 197^{\circ}$ and $200^{\circ} \mathrm{C}$, respectively. Note that the samples were respectively held at these temperatures until no change in heat flow was recorded over time. The kinetics of isothermal crystallization was studied based on the evolved exothermic enthalpy recorded in this isothermal step.

In the non-isothermal crystallization studies, samples were first heated up to $240^{\circ} \mathrm{C}$ at $20^{\circ} \mathrm{C} / \mathrm{min}$ and held for 4 minutes followed by cooling at four different cooling rates of $5^{\circ}, 10^{\circ}, 15^{\circ}$ and $20^{\circ} \mathrm{C} / \mathrm{min}$. The kinetic study is based on exothermic enthalpy evolved during cooling. All the DSC experiments of this study were performed under inert dry nitrogen atmosphere to prevent 
thermo-oxidative degradation of the experimental samples. Thermal stability of the samples was verified by thermogravimetric analyses runs prior to DSC experiments.

\subsection{Solid State ${ }^{13} \mathrm{C}$ NMR}

${ }^{13} \mathrm{C}$ NMR spectra were obtained using a Bruker AVANCE I 400 (9.6 T) NMR instrument using a $4 \mathrm{~mm}$ broadband MAS Probe. Samples were packed in $4 \mathrm{~mm}$ rotors. All spectra were obtained at room temperature and spinning at $6 \mathrm{kHz}$ for ${ }^{13} \mathrm{C}$. The CPMAS T1 filter NMR spectra were obtained using a $5 \mathrm{~s}$ recycle delay, a CP contact time of $2 \mathrm{~ms}$, and $\mathrm{T} 1$ delay of $5 \mathrm{~s}$ to emphasize rigid crystalline regions with longer relaxation times (tens of seconds) and to filter out amorphous mobile regions with short relaxation times ( < one second). The delay times were chosen as optimal and found to give reproducible results. The ${ }^{13} \mathrm{C}$ chemical shift was referenced to the carbonyl resonance $(\delta=176 \mathrm{ppm})$ of the secondary standard glycine with respect to TMS $\delta=$ $0.0 \mathrm{ppm}$.

\section{Results and discussion}

\subsection{Morphology of PA6/CNC nanocomposites}

The observed changes in the morphology and microstructure of the samples as a function of the $\mathrm{CNC}$ surface functionalization are discussed to understand the influence of the latter on the observed crystallization behavior of the nanocomposites. Figure 1 shows the SEM images of the cryo-fractured surfaces of the neat PA6 and the nanocomposites containing 3\% of CNC and APSCNC.
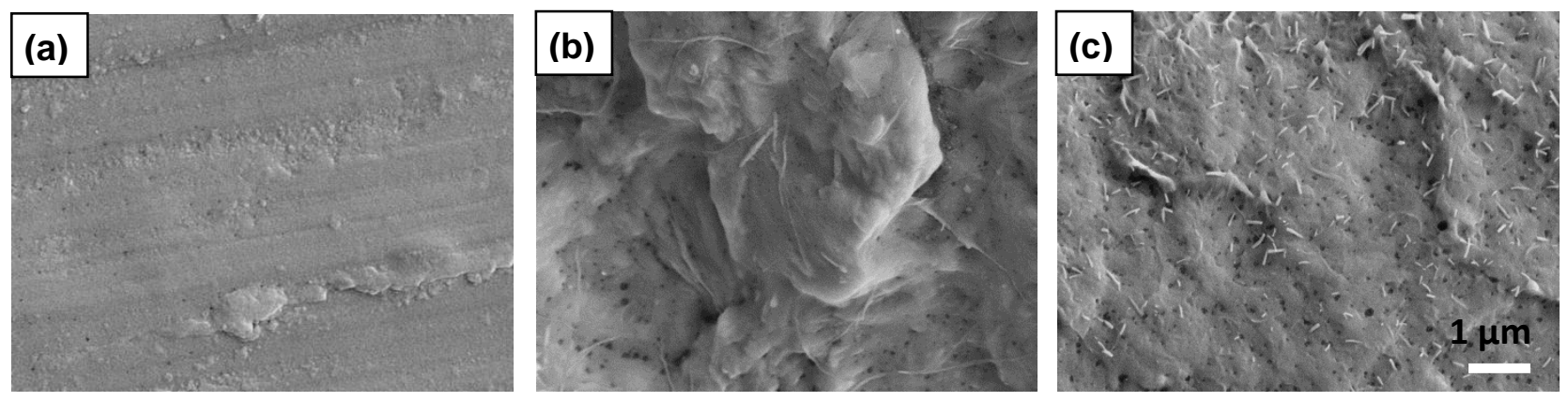

Figure 1: SEM images of the cryo-fractured surfaces of (a) PA6, (b) 3\%CNC and (c) 3\%APS$\mathrm{CNC}$ nanocomposites samples 
Clearly, this figure shows that the neat PA6 matrix shows a featureless surface morphology depicted in Figure 1(a). In the case of 3\%CNC sample, it is evident that the whisker-like cellulose nanocrystals (CNCs) form a microfibrillar-type structure where the bundles of the CNC comprising of interconnected whiskers through edge-to-edge interaction are dispersed in the matrix with a 3D network structure. In a previously reported study on these PA6/CNC nanocomposites systems [27], we observed the network structure development in these systems using melt rheological techniques. Here, it is seen that in the case of the APS-surface modified CNC (or APS-CNC), the dominant morphology is a dispersion of the individual whiskers homogeneously dispersed at the nano-scale within the matrix (see Fig. 1(c)). The observed superior dispersion of the APS-CNC in the PA6 is ascribed to excellent interfacial compatibility with the PA6 matrix due to the presence of amino groups on the APS-CNC surface. The interfacial bond formation is expected to proceed through transamidation reaction between the amide bond on the PA6 chain and the surface amino group of APS layer at the elevated processing temperatures used for sample preparation [29, 30]. A similar type of morphology was observed for the samples containing 1 and $2 \%$ of both CNC and APS-CNC (not shown). The relationship between these observed micro-structural features and the observed crystallization behavior is discussed in detail in the current article.

\subsection{Thermal properties of the melt processed samples}

The melting behavior of the melt processed samples was studied via similar simulated thermal history in the DSC in both the first and second heat runs (the heating and cooling rates were both $10^{\circ} \mathrm{C} / \mathrm{min}$ ) in order to obtain the crystallinity data of the processed samples. The obtained data from both heating runs are shown in Figure 2.
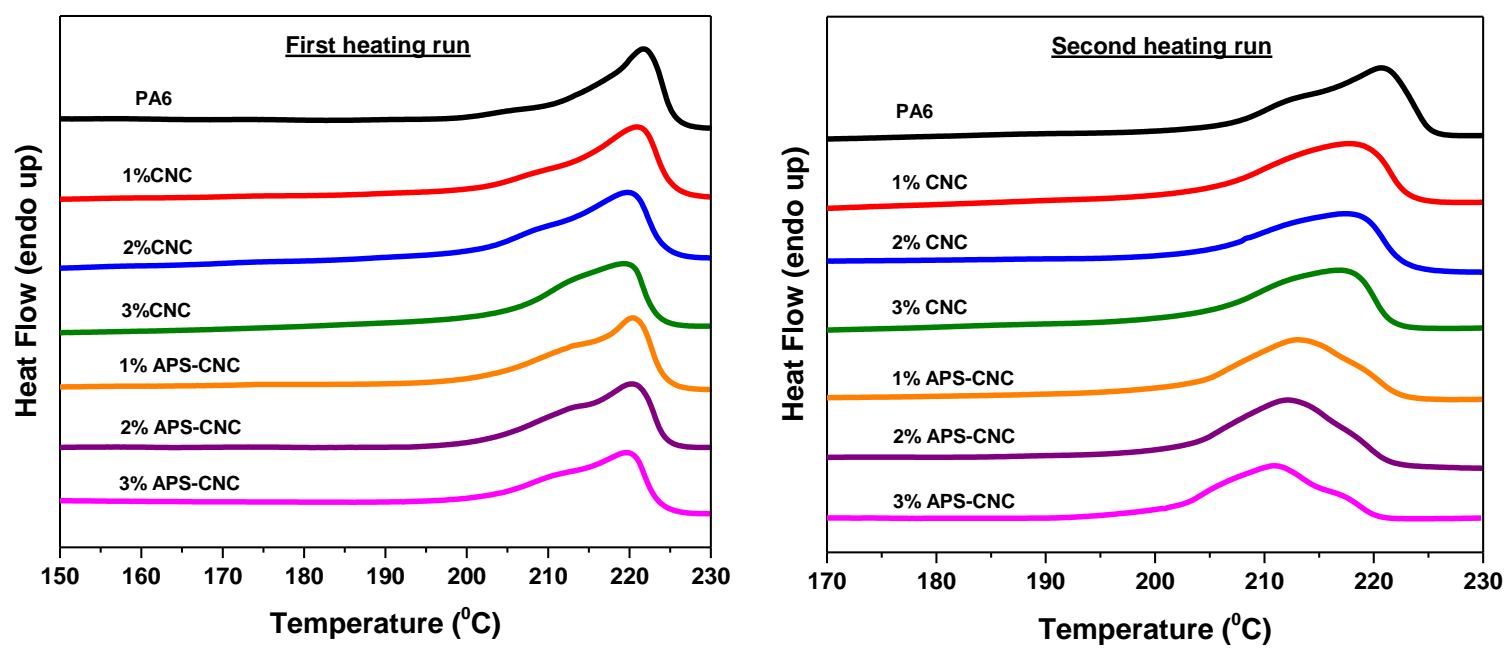

Figure 2: First and second DSC heating thermograms of PA6 and its nanocomposite samples 
From these figures, the melting points and degree of crystallinity were extracted from an average of at least 3 replicates and the obtained results are summarized in Table 1. It is known that pure PA6 has two major crystalline forms, namely the $\alpha$ - and the $\gamma$-crystalline forms where the more thermodynamically stable $\alpha$-form is comprised of monoclinic cell with a relatively more ordered structure and higher melting point compared to that of the less ordered $\gamma$-form with relatively lower melting point. It can be seen in Table 1 that in the first heating runs, the predominant crystal structure of PA6 samples and that of 1 and 2\% of CNC are $\alpha$-type crystals while a portion of $\gamma$-type crystals are observed to develop in the samples with $3 \% \mathrm{CNC}$ and $3 \%$ APS-CNC.

Table 1: Thermal properties of the PA6 and nanocomposites of PA6/CNC and PA6/APS-CNC in first and second heating runs*

\begin{tabular}{ccccccccc}
\hline Sample & \multicolumn{3}{c}{ First heat run } & \multicolumn{5}{c}{ Second heat run after cooling at 10 ${ }^{\circ}$ /min } \\
\cline { 2 - 9 } & $\mathrm{T}_{\mathrm{m} 1}\left({ }^{\circ} \mathrm{C}\right)$ & $\mathrm{T}_{\mathrm{m} 2}\left({ }^{\circ} \mathrm{C}\right)$ & $\Delta \mathrm{H}_{\mathrm{m}}(\mathrm{J} / \mathrm{g})$ & $\chi_{\mathrm{c}}(\%)$ & $\mathrm{T}_{\mathrm{m} 1}\left({ }^{\circ} \mathrm{C}\right)$ & $\mathrm{T}_{\mathrm{m} 2}\left({ }^{\circ} \mathrm{C}\right)$ & $\Delta \mathrm{H}_{\mathrm{m}}(\mathrm{J} / \mathrm{g})$ & $\chi_{\mathrm{c}}(\%)$ \\
PA6 & $222.4(0.4)$ & - & $55.1(1.5)$ & 29.0 & $218.6(0.2)$ & - & $52.6(0.4)$ & 27.7 \\
\hline 1\%CNC & $221.1(0.3)$ & - & $53.4(0.9)$ & 28.1 & $218.1(0.2)$ & - & $48.4(1.2)$ & 25.7 \\
2\%CNC & $219.8(0.1)$ & - & $48,8(0.4)$ & 25.7 & $218.1(0.3)$ & - & $45.1(1.1)$ & 24.2 \\
3\%CNC & $219.2(0.4)$ & - & $44.5(0.2)$ & 23.4 & $217.3(0.5)$ & - & $42.6(0.6)$ & 23.1 \\
\hline 1\%APS-CNC & $221.2(0.2)$ & - & $53.6(0.4)$ & 28.2 & $219.1(0.3)$ & $213.3(0.4)$ & $50.8(0.7)$ & 27.0 \\
2\%APS-CNC & $220.8(0.3)$ & - & $50.8(0.6)$ & 26.8 & $219.2(0.8)$ & $212.2(0.2)$ & $48.3(0.2)$ & 25.9 \\
3\%APS-CNC & $220.1(0.5)$ & - & $48.2(0.4)$ & 25.3 & $217.3(0.5)$ & $211.1(0.1)$ & $45.1(0.4)$ & 24.5 \\
\hline
\end{tabular}

${ }^{*} \mathrm{~T}_{\mathrm{m} 1}$ and $\mathrm{T}_{\mathrm{m} 2}$ respectively denote melting temperatures of $\alpha$ - and $\gamma$-type crystals. Values in parenthesis are standard deviation.

Interestingly, in the second heat runs all the samples including the neat PA6 reveal a certain amount of $\gamma$-type crystals with a relatively very large portion of this $\gamma$-crystalline structure that appears to be the dominant crystal form in the case of APS-CNC nanocomposite samples. Note that an $\alpha$-type crystal is evident as a shoulder peak in the low melting point range of the $\gamma$-type crystal melting point. In other to further verify this observed change in the characteristics of the crystalline structure, we performed solid state ${ }^{13} \mathrm{C}$ NMR analysis on the samples and the results obtained are presented in Figure 3. From the structure of the repeating unit of the PA6 polymer, the peak at 43.5 ppm corresponds to the $\mathrm{C} 1$ carbon atom (i.e., nitrogen neighbor) and the peak at around $30 \mathrm{ppm}$ is attributed to the C2 and C3 carbon atoms, while the peaks at 26.5 and $37 \mathrm{ppm}$ are respectively ascribed to $\mathrm{C} 4$ and $\mathrm{C} 5$ carbon atoms. The $\mathrm{C} 1$ and $\mathrm{C} 5$ carbons appear at 43.5 and 37 in $\alpha$-polymorph while the corresponding peaks of these carbons in $\gamma$-polymorph appear at around 40 and 34 ppm [31]. The NMR spectra of these two samples depicted in Fig. 3 clearly shows that in the case of 3\% CNC sample, the major crystalline peaks of $\mathrm{C} 1$ and $\mathrm{C} 5$ carbon atoms are appeared at the typical positions of $\alpha$-type crystal. By contrast, in the sample with 3\%APS- 
CNC, two smaller peaks observed at 34 and $40 \mathrm{ppm}$ that are exactly located at the positions of $\mathrm{C} 1$ and C5 of $\gamma$-type crystalline regions. Therefore, it can be concluded that the solid state NMR results conformed well to the observed evolution of $\gamma$-type crystals when the CNC particles are surface modified with APS and found to be consistent with DSC thermograms already discussed.

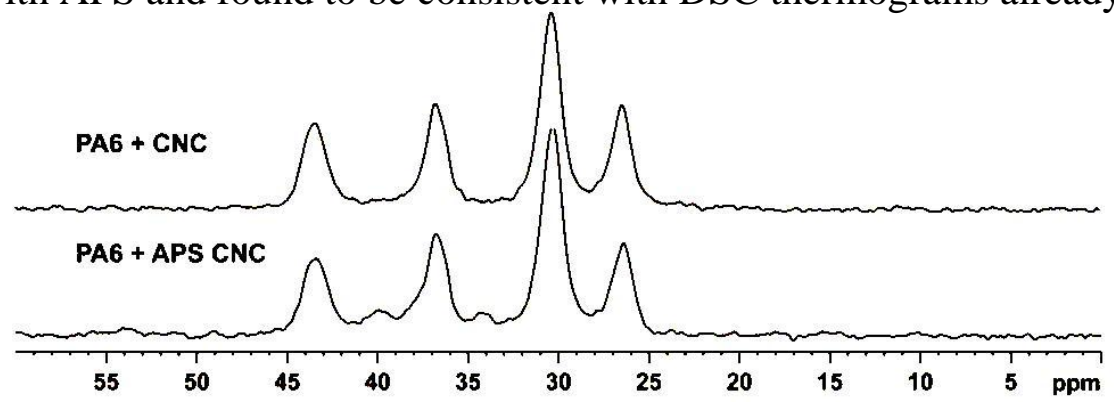

Figure 3: Solid state ${ }^{13} \mathrm{C}$ CPMAS NMR spectra for PA6/3\%CNC and PA6/3\%APS-CNC samples

The obtained DSC data in Table 1 show that the major melting point corresponding to $\alpha$-type ( $T_{\mathrm{m} 1}$ corresponds to $\alpha$-type and $T_{m 2}$ corresponds to $\gamma$-type crystals) of the first heating process decreased as the CNC content was increased, This observation combined with the observed reduction in the overall absolute degree of crystallinity $\left(\chi_{\mathrm{c}}=100 * \Delta H_{\mathrm{m}} /(1-m) \Delta H_{0}\right.$ where ' $m$ ' is the mass fraction of CNC) in the nanocomposite samples suggests that the presence of CNC whisker particles hinders the crystallization of the PA6 matrix chains thereby reducing the overall crystallinity. Note that the major melting points of the crystals are observed to decrease as the CNC content is increased (see Table 1). This observed behavior is consistent with that reported in the literature for high aspect ratio and high performance nano-additives in PA6 samples such as silicates [32] and graphite sheets [33]. A similar trend to the one just described was observed in the case of APS-modified CNC particles with a slight increase in both the $T_{\mathrm{m} 1}$ and degree of crystallinity.

The DSC data obtained from the second heating runs of the samples reveal similar crystallization trend where the observed shoulder peak on the APS-CNC samples (corresponding to the $\alpha$-type crystal) shows a relatively higher melting point compared to that of the non-modified CNC samples (see Table 1). In addition, there is a slight increase in the overall degree of crystallinity in the APS-CNC compared to that of the pristine CNC/PA6 nanocomposite samples. For example, the $3 \%$ APS-CNC composite sample shows $24.4 \%$ of crystallinity compared to a value of $23.1 \%$ for the $3 \% \mathrm{CNC}$ sample. Therefore, it is conjectured that the slight enhancement of crystallinity compared to that of the non-modified $\mathrm{CNC}$ is due to the competition between the promotion of crystallinity that is caused by the surface functionality of APS-CNC and the 
counteracting inhibitive effect on the crystal growth. These results indicate that the APS surface modification of the CNC and the associated improved dispersion of the APS-CNC strongly influence crystallization of the nanocomposites of this study and can, therefore, be used as in the current study to control the crystallization with enhanced benefits. The observed crystallization process in both the isothermal and non-isothermal modes are analyzed in details in the following sections to better understand the role of the effects just mentioned.

\subsection{Theoretical analysis of the observed isothermal crystallization}

In order to study the isothermal crystallization kinetics, the heat release during the process was monitored. A typical thermogram showing the isothermal crystallization exotherm at $194^{\circ} \mathrm{C}$ is shown in Figure 4(a). Clearly, this figure shows that addition of pristine CNC particles tends to move the exotherm towards longer crystallization times. However, an opposite effect is observed in the case of APS modified CNC particles where the crystallization process occurs relatively faster than that of the PA6 sample, suggesting as expected that the surface functionality of the CNC particles greatly changes the isothermal crystallization behavior of PA6/CNC nanocomposites.

The kinetics of isothermal crystallization process can be studied by monitoring the variation of the relative degree of crystalline conversion at a constant crystallization temperature over time. The values of the relative degrees of crystallinity, $\mathrm{X}_{\mathrm{c}}(t)$, can be obtained from the area under the exothermic peak of isothermal crystallization in the DSC thermogram at time $t$ divided by the total area under the exotherm as shown in equation (1)

$$
X_{c}(t)=\frac{\Delta H_{t}}{\Delta H_{\infty}}=\frac{\int_{0}^{t}\left(\frac{d H}{d t}\right) d t}{\int_{0}^{\infty}\left(\frac{d H}{d t}\right) d t}
$$

The $\mathrm{X}(t)$ values can then be converted into the volumetric fractional crystallinity using the following equation [34]:

$$
V_{c}=\frac{X_{c}}{X_{c}+\left(\frac{\rho_{c}}{\rho_{a}}\right)\left(1-X_{c}\right)}
$$

The variation of the volumetric fractional crystallinity is plotted against time (at three different crystallization temperatures) in the Figures 4(b)-(d). These figures show that as the temperature of crystallization increases, the curves are shifted towards higher crystallization times, indicating that 
the rate of crystallization decreases as the temperature is increased from $194^{\circ} \mathrm{C}$ to $200^{\circ} \mathrm{C}$. It is also evident in Figure 4 that the curves follow a sigmoidal "S" shape pattern. Initially, the slope of the curve increases and as the time of crystallization progresses, the slope decreases at the later stages of crystallinity indicating a secondary crystallization process followed by the plateau region corresponding to the crystal impingement.

The Johnson-Mel-Avrami-Kolmogorov (or $J M A K)$ (or simply Avrami) model has been successfully used in order to describe the kinetics of solid state phase transformations [35]. In the context of isothermal crystallization process, this theory describes the time dependence of fractional crystallinity using the following equation (3) [36]:

$$
1-V_{c}(t)=\exp \left(-k t^{n}\right)
$$

where the $\mathrm{V}_{\mathrm{c}}(t)$ is the volumetric fractional crystallinity at time $t . K$ is the Avrami rate constant containing the contribution of both nucleation and growth, and $n$ is the Avrami index which is related to the geometrical features of the spherulitic crystal development and is usually a value between 1 to 4 . Equation 4 is a form of the Avrami equation written in the double logarithmic format as follows:

$$
\log \left[-\ln \left(1-V_{c}(t)\right)\right]=\log K+n \log t
$$

If the Avrami model is applicable to the data, fitting of this model to the data should yield a straight line from which the Avrami exponent and rate constant can be respectively obtained from the slope and intercept of the line. The Avrami fit to the obtained data from PA6, 3\%CNC and $3 \%$ APS-CNC samples are shown in Figure 5 (for all samples, $\mathrm{R}^{2} \geq 0.99$ ). It can be seen from the curves of the Avrami plots that three sections with different slopes can be distinguished; an initial stage from the beginning of the crystallinity until 10-20\% crystallinity followed by a secondary phase with a higher slope that extends to near $75 \%$ crystallinity. This last stage is then followed by a final phase of crystallization with significantly lower slope due to the secondary crystallization and crystal impingement. Interestingly, the shape of the curves is observed to be different in the case of the nanocomposites samples especially those containing the $3 \% \mathrm{CNC}$ and $3 \% \mathrm{APS}-\mathrm{CNC}$. 

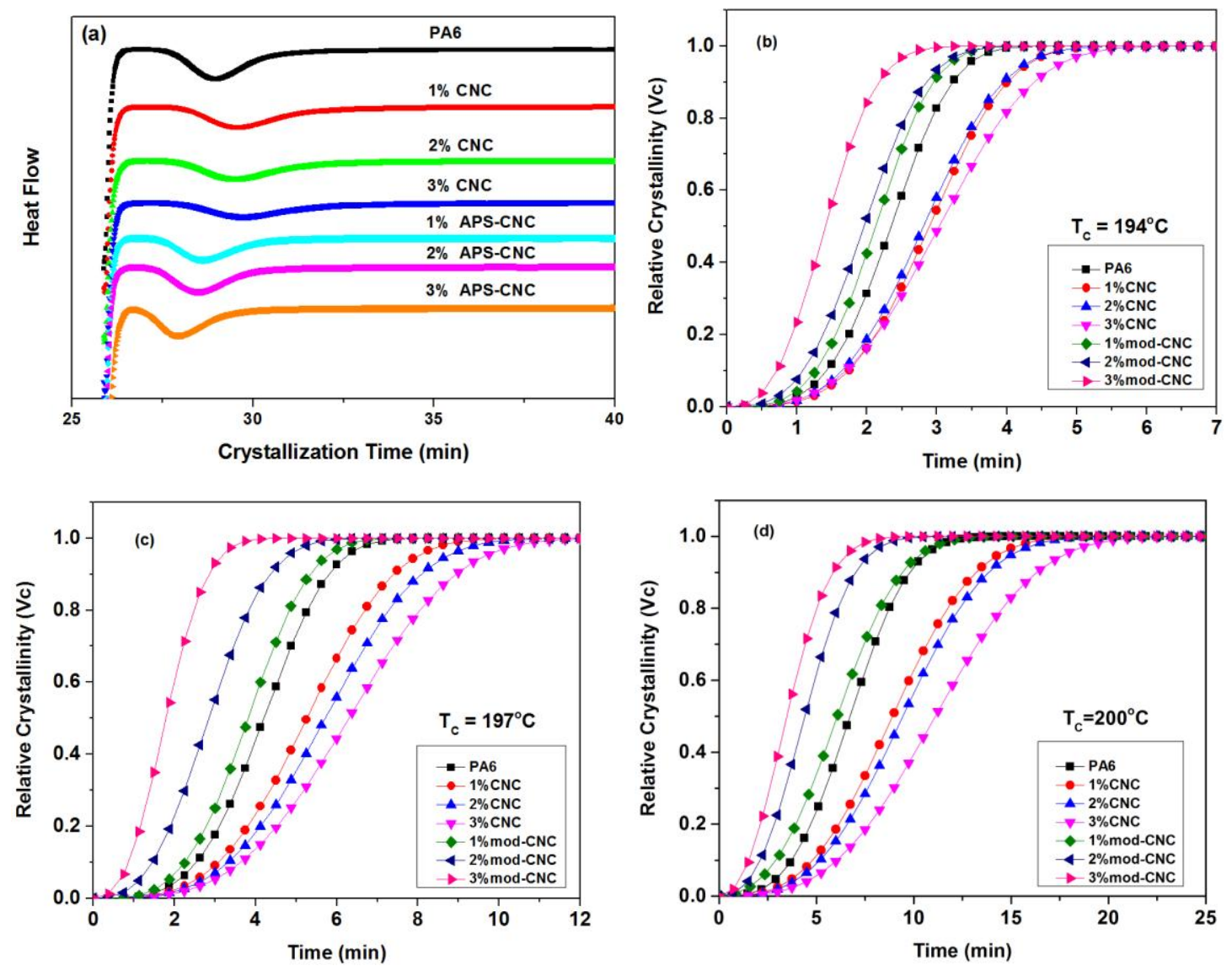

Figure 4: (a) Isothermal crystallization exotherms at $194^{\circ} \mathrm{C}$, variation of relative degree of crystallinity versus time at crystallization temperatures of (b) $194^{\circ} \mathrm{C}$, (c) $197^{\circ} \mathrm{C}$ and (d) $200^{\circ} \mathrm{C}$

Unlike the PA6 sample, the nanocomposites show negligible secondary crystallization indicated by the absence of the distinctive roll-off behavior at the end of the fitted line (later stages of crystallinity). This observed behavior is ascribed to the strong steric hindrance due to the physical presence of the solid CNCs and the strong interaction and interfacial chemical bond (in the case of APS-CNC sample) that restricts the PA6 macromolecular chain movement into crystalline regions.

The Avrami parameters are obtained from the fits to the experimental data and are shown in Table 2. As previously reported by Zhou and coworkers [37] the Avrami exponent " $n$ " is composed of two terms:

$$
n=n_{n}+n_{d}
$$

where $n_{n}$ represents the contribution of the nucleation process in the Avrami constant. The value of $n_{n}$ is either 1 (in the case of homogenous nucleation) or 0 (in the case of heterogeneous nucleation). However, since in most cases, the nucleation process involves both the homogenous 
and heterogeneous nucleation, the value is usually between 0 and 1 which explains the common non-integer Avrami exponent seen in many systems. The $n_{d}$ is related to the morphology and dimension of the crystal growth; and in the case of polymers it usually takes the integers 1,2 or 3 . The number 3 denotes a 3D spherulitic growth while a reduction of the integer to 2 and 1 signal the epitaxial growth of two dimensional lamellar structures or defected spherulites. In addition, the Avrami exponent predicts the dependence of nucleation on time which in turn depends on the heterogeneity of the nucleation.
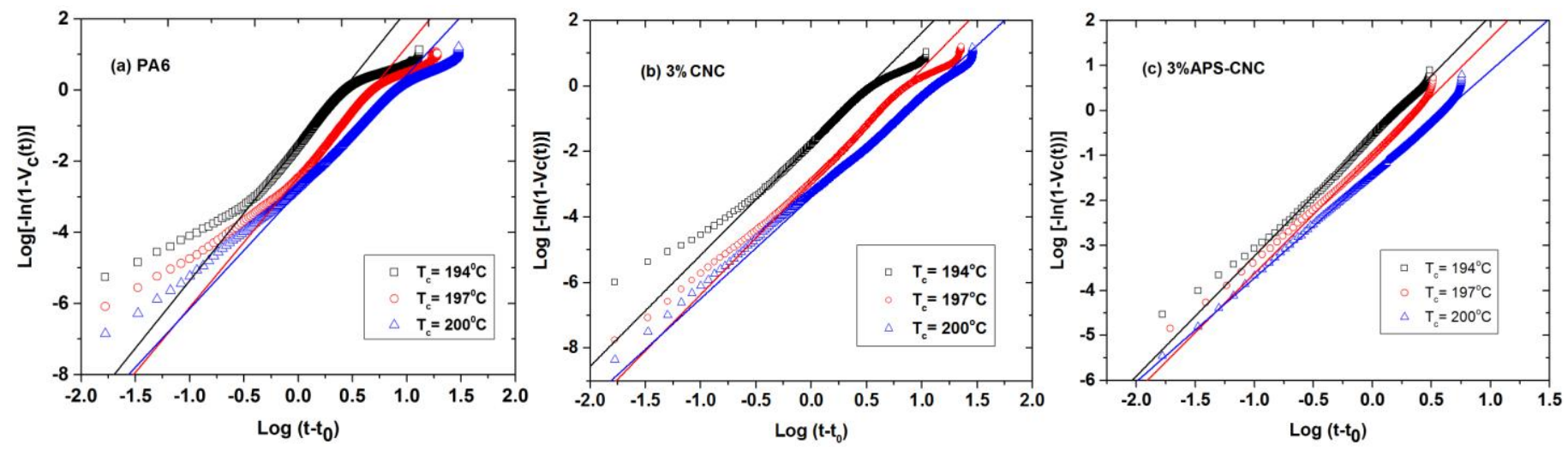

Figure 5: Avrami plots of $\log \left[-\ln \left(1-\mathrm{V}_{\mathrm{c}}(\mathrm{t})\right)\right]$ vs $\log \left(\mathrm{t}-\mathrm{t}_{0}\right)$ for isothermal crystallization of (a)PA6, (b) $3 \% \mathrm{CNC}$ and (c)3\%APS-CNC nanocomposites

Because the nucleation process in filled polymer systems are relatively complicated processes with a combination of both homogenous and heterogeneous nucleation types, the overall Avrami exponent, takes non-integer values as depicted in Table 2. The obtained Avrami exponents are found to vary between 3 and 4 for the neat PA6 and the sample with 3\%CNC, indicating a spherulitic morphology of the crystals. However, the $n$ exponent decreases in the case of samples filled with pristine CNC particles compared to that of the PA6 sample. This shows the evolution of imperfect spherulites that tend towards epitaxial growth (i.e., reduction of $n$ value toward values less than 3).

A similar effect is evident when the $\mathrm{CNC}$ concentration is increased from 1 to $3 \mathrm{wt} \%$, showing that at higher concentration of CNC whiskers, the crystal growth mechanism is confronted with less free volume due to the presence of CNCs and restricted molecular motion. In the case of CNC whiskers with APS surface functionality, the Avrami exponent decreased even further and eventually to values less than 3 in the case of 3\%APS-CNC sample. In a similar analogy, the formation of interfacial bond during the course of polymerization/melt extrusion is conjectured to further restrict the PA6 macromolecular chain motion especially in the interfacial area. In addition, 
the nanoscale dispersion of individual whiskers as seen in the SEM images of this sample already described, suggests that the increased overall interfacial area between the matrix and the surface of the $\mathrm{CNC}$ particles have significant effects on the dimensionality of the crystals leading to the formation of 2D structures with less crystal perfection as already mentioned.

Table 2: Kinetic parameters obtained from Avrami analysis of isothermal crystallization of PA6, PA6/CNC and PA6/APS-CNC nanocomposites*

\begin{tabular}{|c|c|c|c|c|}
\hline Sample & $\mathbf{T}_{\mathrm{c}}\left({ }^{\circ} \mathbf{C}\right)$ & $\mathbf{n}$ & $K^{*} 10^{4}\left(\min ^{-1}\right)$ & $t_{1 / 2}(\min )$ \\
\hline \multirow[t]{3}{*}{ PA6 } & 194 & 3.81 & 275.5 & 2.38 \\
\hline & 197 & 3.66 & 36.3 & 4.25 \\
\hline & 200 & 3.28 & 13.8 & 6.77 \\
\hline \multirow[t]{3}{*}{$1 \% \mathrm{CNC}$} & 194 & 3.71 & 135.1 & 2.93 \\
\hline & 197 & 3.53 & 19.7 & 5.31 \\
\hline & 200 & 3.12 & 8.4 & 9.02 \\
\hline \multirow[t]{3}{*}{$2 \% \mathrm{CNC}$} & 194 & 3.54 & 187.4 & 2.87 \\
\hline & 197 & 3.49 & 15.7 & 5.75 \\
\hline & 200 & 3.15 & 5.8 & 9.51 \\
\hline \multirow[t]{3}{*}{$3 \% \mathrm{CNC}$} & 194 & 3.39 & 187.7 & 3.13 \\
\hline & 197 & 3.44 & 12.3 & 6.39 \\
\hline & 200 & 3.09 & 4.1 & 11.18 \\
\hline \multirow[t]{3}{*}{ 1\%APS-CNC } & 194 & 3.66 & 438.1 & 2.15 \\
\hline & 197 & 3.48 & 55.5 & 3.86 \\
\hline & 200 & 2.98 & 23.6 & 6.73 \\
\hline \multirow[t]{3}{*}{$2 \%$ APS-CNC } & 194 & 3.22 & 794.7 & 1.98 \\
\hline & 197 & 2.93 & 356.2 & 2.87 \\
\hline & 200 & 2.68 & 147.7 & 4.43 \\
\hline \multirow[t]{3}{*}{ 3\%APS-CNC } & 194 & 2.67 & 2754.2 & 1.42 \\
\hline & 197 & 2.62 & 1000.3 & 1.78 \\
\hline & 200 & 2.30 & 380.2 & 3.45 \\
\hline
\end{tabular}

*The reported crystallization half-times $\left(\mathrm{t}_{1 / 2}\right)$ are experimental values.

Another important parameter in the Avrami equation is the Avrami crystallization parameter or $K$ which is related to both the nucleation and growth rate $[14,38]$. The current study shows that with increasing temperature of crystallization this parameter decreases, implying a relatively slower overall nucleation and growth rate at typical elevated temperatures. However, with addition of the CNC whiskers, opposite effects are observed in samples containing pristine CNC and APS$\mathrm{CNC}$ samples. Specifically, the pristine CNC particles tend to decrease the $K$ values, indicating a slower nucleation and growth rate while the APS modified CNCs increase the $K$ value (see Table 2). This observed experimental fact that the APS modified CNC particles increase the nucleation and crystal growth rate is thought to be related to the occurrence of heterogeneous nucleation where, in fact, in these samples the solid CNC whiskers act as effective nucleating agents. Because there is a competing effect of both the nucleation process and crystal growth rate retardation, it is evident that in the case of the samples containing pristine $\mathrm{CNC}$ particles, the crystal growth rate 
retardation is the dominating effect especially considering the fibrillar network-like microstructure in these samples as shown in the SEM images already described. For example, at lower temperature when the nucleation is faster than crystal growth, the $K$ value shows a slight increase as the CNC content increases from 1 to $3 \mathrm{wt} \%$ while it remains smaller than that of the neat PA6. In a previously reported study on the non-surface modified CNC/PA6 nanocomposite systems [27] we showed that the viscosity of the PA6 matrix can be significantly increased with a very small volume fraction of CNC whiskers. Therefore, the increased viscosity can clearly slow down the transfer of new chains into the growing crystals. On the other hand, in the case of APS-modified samples, the crystal nucleation effect is more pronounced as indicated by an increase in the $K$ value while the lower Avrami exponent (crystal growth dimensionality) shows significant restrictions on crystal growth. Consequently, it can be argued that while the APS-CNC particles tend to promote nucleation of crystals, they also can impose a crystal growth hindrance at the same time. These overall competing effects are deconvoluted and explained further later in more detail in the theoretical analysis of the non-isothermal crystallization data.

Another parameter shown in Table 2 is the crystallization half-time or $t_{1 / 2}$ which is the experimental time required to reach $50 \%$ relative crystallinity. The data presented in this table shows that the crystallization half-time increase with increasing temperature, indicating relatively slower crystallization rates at elevated temperatures. With the addition of the pristine $\mathrm{CNC}$, the crystallization half-time increased compared to that of the neat PA6, and decreased further as the CNC concentration was increased. This observation shows that the overall nucleation and growth phenomena are taking place at relatively lower rates when the non-modified CNC whiskers are incorporated into the matrix. By contrast, the samples containing the APS-CNC particles show a decrease in the crystallization half-time that again confirms the significant heterogeneous nucleating effect of these particles that outweighs the crystal growth retardation effect of particles especially at earlier stages of crystallinity as already described.

The data obtained from the isothermal crystallization experiments can be evaluated in the context of Lauritzen-Hoffman ( $\mathrm{LH})$ crystal growth theory $[39,40]$ to obtain comparative kinetic parameters of crystal nucleation and growth process for the neat PA6 with that of nanocomposites with $3 \mathrm{wt} \%$ of pristine CNC and APS-CNC. According to this LH secondary nucleation theory there are two major processes that govern the crystal growth. These processes are the secondary nuclei deposition followed by polymer chain transport and lateral growth along the face where the secondary nuclei were established. Depending on the relative rates of secondary nuclei formation 
(i) and lateral growth rate $(g)$, three different regimes exist where $i<<g$ (regime I), $i \sim g$ (regime II), and $i>>$ (regime III).

The growth rate of the crystal can be approximated with the reciprocal crystallization half-time as $G=\left(t_{1 / 2}\right)^{1 / 2}$ where the half-time was calculated from the isothermal crystallization exotherms as already discussed in the previous section. Subsequently, the energetics of the process can be described using the Hoffman equation as follows:

$$
G=G_{0} \exp \left[-\frac{U^{*}}{R\left(T_{C}-T_{\infty}\right)}\right] \exp \left[-\frac{K_{g}}{T_{C} \Delta T f}\right]
$$

where the $G_{0}$ is the pre-exponential term, $U^{*}$ is the diffusional activation energy for the transport of the crystallizable segments at the melt-crystal interface with a universal value of $1500 \mathrm{cal} / \mathrm{mol}, T_{C}$ is the crystallization temperature, $T \infty$ is the temperature below which the viscous flow of the polymer ceases which is commonly taken as $\left(T_{\mathrm{g}}-30^{\circ} \mathrm{K}\right), f$ is correction factor to account for the change in the enthalpy of fusion of perfect crystal with the temperature which is taken as $2 T_{\mathrm{C}} /\left(T_{\mathrm{m}}{ }^{0}+T_{\mathrm{C}}\right)$ where the $T_{\mathrm{m}}{ }^{0}$ is the equilibrium melting point, $\Delta T$ is defined by $T_{\mathrm{m}}{ }^{0}-T_{\mathrm{C}}, K_{\mathrm{g}}$ is the nucleation parameter that is related to the necessary free energy to form critical sized nucleus, and $R$ is the gas constant. In this study, the Hoffman-Weeks approach [41] was adopted to find the equilibrium melting point of the neat PA6 and the 3\% nanocomposite samples containing pristine CNC and APS-modified CNC that were crystallized at four different temperatures of $194^{\circ}, 197^{\circ}$, $200^{\circ}$ and $203^{\circ} \mathrm{C}$, and subsequently heated above the corresponding melting point. The obtained Hoffman-Weeks plot is shown in Figure 6(a). The mathematical relationship between the crystallization temperature, melting temperature and the equilibrium melting temperature is described according to the following equation (7) [14]:

$$
T_{m}=T_{m}^{0}\left(1-\frac{1}{\gamma}\right)+\frac{T_{C}}{\gamma}
$$

where the $\gamma$ is a parameter related to the final lamellar thickness. In this plot, a straight line is fitted on the $T_{\mathrm{m}}$ versus $T_{\mathrm{C}}$ data and the intersection with the $T_{\mathrm{m}}=T_{\mathrm{C}}$ line gives the equilibrium melting point as shown in the insert data of Figure 6(b). 

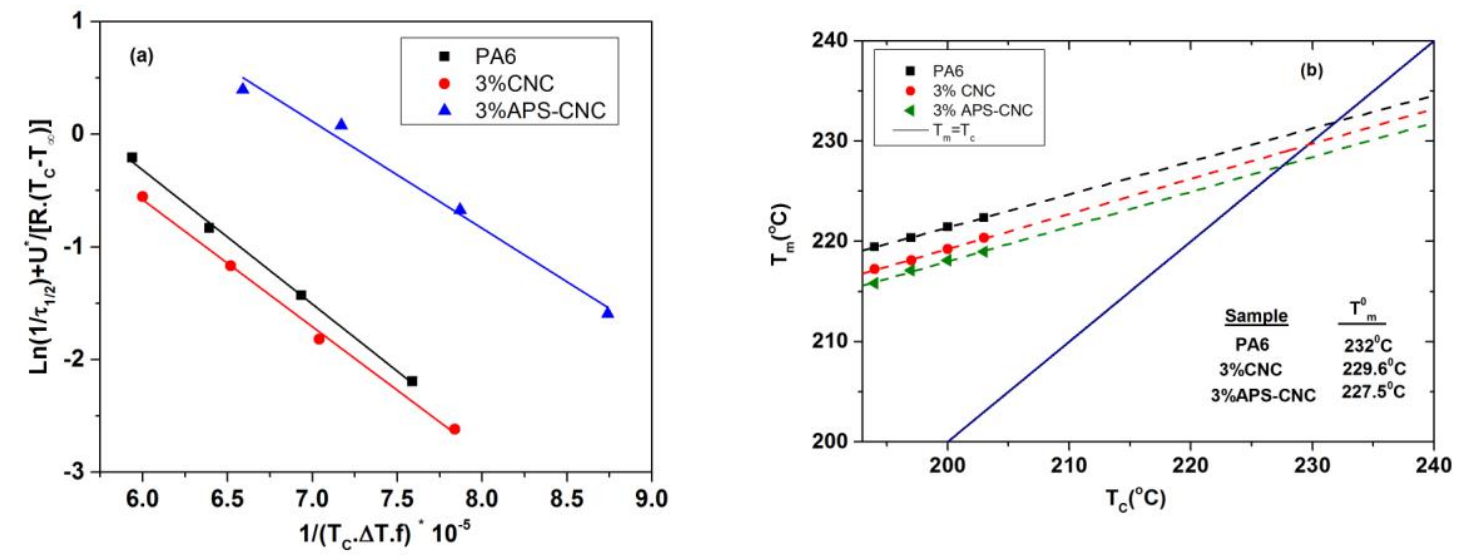

Figure 6: (a) Lauritzen-Hoffman plot and (b) Hoffman-Weeks plot for PA6, $3 \% \mathrm{CNC}$ and 3\%APS-CNC nanocomposites

The obtained equilibrium melting points for the PA6, 3\% CNC and the 3\% APS-CNC are $231.9^{\circ}$, $229.6^{\circ}$ and $227.6^{\circ} \mathrm{C}$ as indicated in the Figure $6(\mathrm{~b})$. It should be noted that for the APS-CNC system, the second melting point corresponding to the $\alpha$-type crystals are considered. The obtained values for the equilibrium melting points are in good agreement with those previously reported for PA6-based systems [14, 33, 42].

Now using the obtained $T_{\mathrm{m}}{ }^{0}$ values obtained, the Hoffman equation can be re-arranged in the following format:

$$
\ln G+\frac{U^{*}}{R\left(T_{C}-T_{\infty}\right)}=\ln G_{0}-\frac{K_{g}}{T_{c}(\Delta T) f}
$$

This equation is plotted for the neat PA6, 3\%CNC and 3\%APS-CNC samples in Figure 6(a) that shows a good linear fit to the experimental data; and from the slope of the lines the nucleation parameter (or $K_{\mathrm{g}}$ ) can be obtained. This $K_{\mathrm{g}}$ parameter contains contributions from the folded and lateral surface energies according to the following equation (9) [23]:

$$
K_{g}=\frac{m b \sigma \sigma_{e} T_{m}^{0}}{\Delta H_{f}^{0} \cdot k_{B}}
$$

where $b$ is the distance between the two adjacent fold planes which in our case corresponds to the $\alpha$-type crystal with a monoclinic cell structure with lattice parameters of $(0.956 \mathrm{~nm} \times 0.801 \mathrm{~nm} \mathrm{x}$ $1.72 \mathrm{~nm})$ [16], $\sigma$ and $\sigma_{\mathrm{e}}$ are respectively the lateral and folded surface energies, $\Delta H_{\mathrm{f}}{ }^{0}$ is the enthalpy of fusion of a perfect crystal of PA6 equal to $190 \mathrm{~J} / \mathrm{g}$ [43], $k_{B}$ is the Boltzman constant and the $m$ is 
an integer with a value of 2 in the case of regime II and 4 in the case of regimes I and III. Here, a value of $m=2$ is used as will be shown later that the obtained data correspond to the regime II of crystallization using a Z-test. However, it should be noted that the unit cell values are assumed to be similar because the content of $\mathrm{CNC}$ is low in the PA6 matrix like other researchers have reported in the literature in a number of previous studies [14, 43]. From the values of $\sigma$ and $\sigma_{\mathrm{e}}$, the work of chain folding denoting the energetics of the crystallizing chain to fold back into the lamella is obtained using the following expression:

$$
q=2 a b \sigma_{e}
$$

The results and values of this analysis are tabulated in the Table 3. As can be seen from this table, the value of the nucleation constant slightly increases in the case of $3 \% \mathrm{CNC}$ samples which correlates with the longer half times obtained in the Avrami analysis already described while for the $3 \%$ APS-CNC the $K_{\mathrm{g}}$ values is significantly decreased. The slight increase in the case of $3 \% \mathrm{CNC}$ is somewhat contradictory to what one would expect from a secondary nucleation role of a solid surface as a number of previous studies have shown that clay [8] and carbon nanotubes [38] nanoparticles can act as effective nucleating agents in PA6 matrix thereby promoting secondary nuclei formation.

Table 3: Parameters of Hoffman-Lauritzen analysis of isothermal crystallization of PA6, PA6/CNC and PA6/APS-CNC nanocomposites*

\begin{tabular}{cccc}
\hline & PA6 & 3\% CNC & 3\%APS-CNC \\
\hline $\mathbf{K}_{\mathbf{g}} * \mathbf{1 0} \mathbf{1 0}^{-\mathbf{5}}\left(\mathbf{K}^{\mathbf{2}}\right)$ & 1.17 & 1.25 & 0.95 \\
$\boldsymbol{\sigma}\left(\mathbf{m J} / \mathbf{m}^{\mathbf{2}}\right)$ & 30 & 30 & 30 \\
$\boldsymbol{\sigma}_{\mathbf{e}}\left(\mathbf{m} \mathbf{J} / \mathbf{m}^{\mathbf{2}}\right)$ & 7.46 & 7.87 & 5.91 \\
$\mathbf{q}(\mathbf{J}) * \mathbf{1 0}^{\mathbf{2 0}}$ & 2.46 & 2.62 & 1.91 \\
\hline
\end{tabular}

It has also been reported in the literature that $\mathrm{CNC}$ whiskers can effectively promote the nucleation of crystals in poly(caprolactone) [23] and PLA [44]. On the other hand, in a recent study by Guo et al [16] the Lauritzen-Hoffman analysis on a PA6 system reinforced with halloysite ${ }^{\circledR}$ nanotubes showed an increase in the $K_{g}$ parameter with addition of the nanoparticles which was interpreted to be related to the inhibitive effect of strong interactions between the nanotube surface and PA6 chains. In the current PA6/CNC nanocomposite system, it is conjectured that the strong hydrogen bonding interaction between the PA6 and the hydroxyl-rich surface of cellulose whiskers and the microfibrillar microstructure that was developed in the case of non-treated CNC imposes a significant restriction on the molecular motion of the PA6 chains 
that results in the slow-down in the crystallization process. This explanation is consistent with a previously reported study on these systems [27] that shows that the viscous flow of the PA6 chains both in solid-state and melt-state is significantly reduced in the presence of the CNC whiskers, thus justifying the increase in $K_{g}$ parameter.

In contrast to the pristine CNC, the APS-CNC particles used in this study showed a much finer phase distribution within the PA6 matrix, resulting in much higher available surface area. Additionally, the interfacial bond formation with the amine groups on the APS-CNC surface results in enhanced surface activity for crystal nucleation process as indicated by the lower $K_{\mathrm{g}}$ value. By considering the $\sigma_{\mathrm{e}}$ values, it is seen that in the case of $3 \% \mathrm{CNC}$, the value is increased from $7.46 \mathrm{~mJ} / \mathrm{m}^{2}$ to $7.84 \mathrm{~mJ} / \mathrm{m}^{2}$ suggesting a less energetically favorable folded surface in the lamella. On the other hand, the folded surface energy is reduced to $5.91 \mathrm{~mJ} / \mathrm{m}^{2}$ in the case of the $3 \%$ APS-CNC sample, suggesting a lower energy barrier of crystallization process that is facilitated by the modified surface chemistry and more homogenous dispersion of whiskers in the matrix. As already described in the Avrami analysis, even the APS-CNC samples showed a significant crystal growth restriction on the crystals which was verified by the reduction in the obtained Avrami constants to values lower than 3. This observed effect in the current analysis can be discussed using the work of chain folding $(q)$ parameter given in Table 3 that shows that the $q$ value is increased from $2.4 * 10^{-20} \mathrm{~J}$ to $2.6^{*} 10^{-20} \mathrm{~J}$ in the case of $3 \% \mathrm{CNC}$. This finding suggests a relatively more difficult chain folding process in the presence of $\mathrm{CNC}$ whiskers compared to that of the APS-CNC that showed a decrease in $q$ value to $1.9 * 10^{-20} \mathrm{~J}$ in the $3 \%$ APS-CNC sample (see Table 3). Although this last result may be interpreted as less work required for the chains to fold back into the lamella, it is believed that the reason for this reduction is due to the fact that the crystals in 3\%APS-CNC sample are smaller and less perfect in shape (i.e., distorted crystal structure) that makes it "easier" for the chains to fold back into the crystal.

In summary of the important points of the foregoing discussion, the results show that in the case of pristine $\mathrm{CNC}$ whiskers the crystallization rate is reduced due to the significant restriction imposed on the molecular motion of PA6 chains. Considering the competition between the heterogeneous nucleation activity and the restriction of crystal growth, the obtained results indicate that effect of the latter outweighs that of the former. In the case of APS-modified CNC particles, the nucleation activity is more significant and the reduction in crystal growth is balanced out by the fact that the crystals are less perfect, making the energetics of crystal growth relatively more favorable as will be further verified in the next section using the optical micrograph images. 
Interestingly, in the reported study by Siqueira and co-workers [23], it was shown that microfibrillated cellulose (MFC) imposed more restriction on PCL crystal growth and limited the crystal dimensions, in contrast to the cellulose whiskers that acted as effective nucleating agents with less crystal growth hindrance effect due to the absence of entangled cellulose structures as in the case of MFC which is consistent with the findings of this current study.

Now in order to verify that the crystallization process is indeed in the regime II of HoffmanLauritzen analysis, a Z-test was carried out using the following equation [45]:

$$
Z \approx 10^{3} \cdot\left(\frac{L}{2 a_{0}}\right)^{2} \cdot \exp \left(-\frac{X}{T_{c} \Delta T}\right)
$$

where $L$ is the effective lamellar width, $a_{0}$ is the width of the molecular chain in crystal. If the regimes I or III are applicable, the replacement of $X$ with $K g$ should yield $Z \leq 0.01$. Conversely, if the regime II is applicable, the replacement of $X$ with $2 K \mathrm{~g}$ should yield $Z \geq 1.0$. This Z-test used the values of $K_{\mathrm{g}}$ already described in equation (9) and an estimated $L$ value to check whether or not the latter value is realistic. By using a chain width $\left(a_{0}\right)$ value of $0.37 \mathrm{~nm}$ for the PA6 chain with the thickness of a monomolecular layer $\left(b_{0}\right)$ of $0.44 \mathrm{~nm}$ [14], in the first scenario, the equation (11) yields $L \leq 0.12 \mathrm{~nm}$ which is clearly not realistic. Using the second regime approximation, the equation yields $L \geq 6.6 \mathrm{~nm}$ which is a realistic value for the PA6, therefore, confirming the assumption of the second regime of the crystallization in the Hoffman analysis already discussed

To confirm the validity of the hypothetical crystal geometry used in the above Hoffman analysis, cross-polarized optical microscopic images (or POM images) were collected during the isothermal crystallization process. For this purpose, the POM images taken at early stage of crystallization or the nucleation phase and in the state of fully gown crystals after completion of the isothermal crystallization and cooling to room temperature are shown in Figure 7. For the early stage of crystallization, the time required to reach less than 15-20\% crystallinity was chosen from the obtained DSC data. Unlike a previous study by Katoh et al [46] where POM images of PA6 based nanocomposite samples were taken at similar time intervals for all samples, we took the POM images at similar "crystalline content" among the samples to have a better observation of the individual samples and their crystalline morphology. As can be seen in the POM images, for the neat PA6 sample a full Maltese cross and a spherulitic morphology with positive birefringence can be observed. In the case of 3\% CNC sample, a remarkably similar type of morphology with a 
relatively more diffuse Maltese cross patterns is observed, indicating formation of defected spherulites and major linear-shaped crystalline regions that shows the hindrance imposed by CNCs on spherulite development. By contrast, in the case of 3\%APS-CNC sample, it is clearly seen that a greater number of nuclei formed at early stage of crystallization which indicates crystal nucleation property of the CNC whiskers as already mentioned. However, the size of the crystals and spheres are observed to be relatively much smaller compared to that of the PA6 spherulites even for the sample containing the highest CNC concentration (i.e., 3\%CNC) studied. These results strongly indicate that the surface modified CNC particles exhibit significant heterogeneous nucleating effect while largely restricting the spherulite growth in 3 dimensions. In fact, enhanced dispersion of APS-CNC in the matrix together with the higher surface activity is resulting in formation of relatively large number of nuclei with larger number of smaller spheres and underdeveloped morphology.
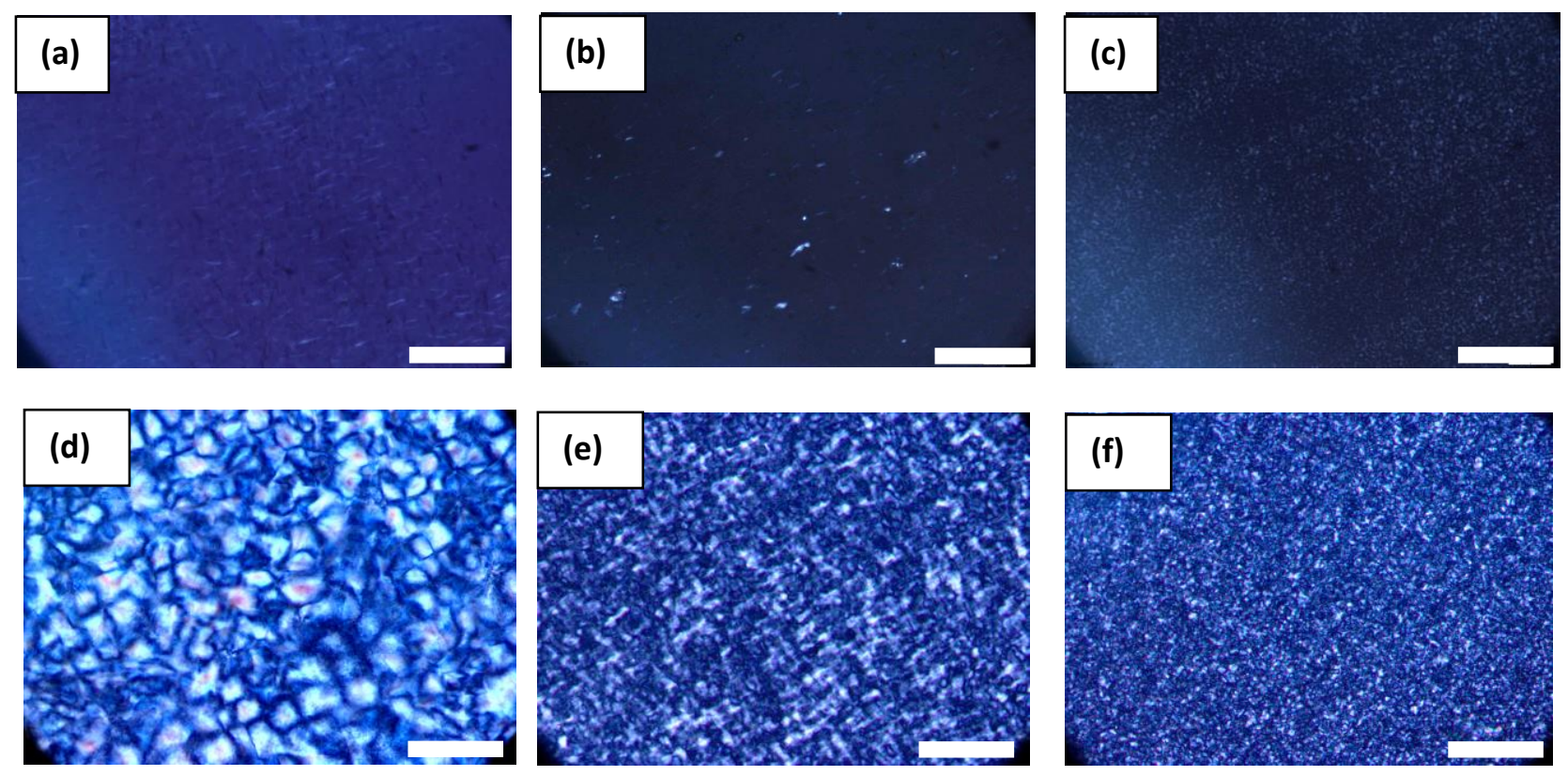

Figure 7: POM images during the early stages of crystallization (nucleation) of (a) PA6, (b) $3 \% \mathrm{CNC}$ and (c)3\%APS-CNC and the final morphology of the developed crystals after isothermal crystallization and cooling to room temperature for (d) PA6, (e) 3\%CNC and (f)3\%APS-CNC samples (scale bar is $50 \mu \mathrm{m}$ )

\subsection{Theoretical analysis of obtained non-isothermal crystallization data}

In order to better understand and interpret the effects of microstructure and surface functionality on the crystallization behavior of the PA6/CNC nanocomposite systems, a series of non-isothermal crystallization experiments were conducted on PA6, PA6/3\%CNC and 
PA6/3\%APS-CNC samples. The samples were cooled at controlled cooling rates of $5^{\circ}, 10^{\circ}, 15^{\circ}$ and $20^{\circ} \mathrm{C}$ from the melt and the exotherms of the crystallization were monitored. The obtained exothermic peaks of crystallization at different rates are shown in Figure 8 with corresponding peak crystallization temperatures reported in Table 4. Clearly, Figure 8 and Table 4 show that increasing the cooling rate results in a decrease in the peak crystallization temperatures for all samples, and in addition, the onset of crystallization temperature occurs at lower temperatures and the crystallization exotherms become broader. In fact, as the cooling rate increases, the available response time of the polymer chains in order to overcome the nucleation energy barrier becomes shorter. Therefore, the crystallization occurs at preferably lower temperature since at lower temperatures, the nucleation rate is enhanced.

Due to the observed lower temperatures of crystallization and associated restriction of the macromolecular motion, the packing of the polymer chains into crystalline regions occurs in a relatively larger time span that results in the obtained crystallization exotherms becoming broader [47]. Comparing the data obtained from

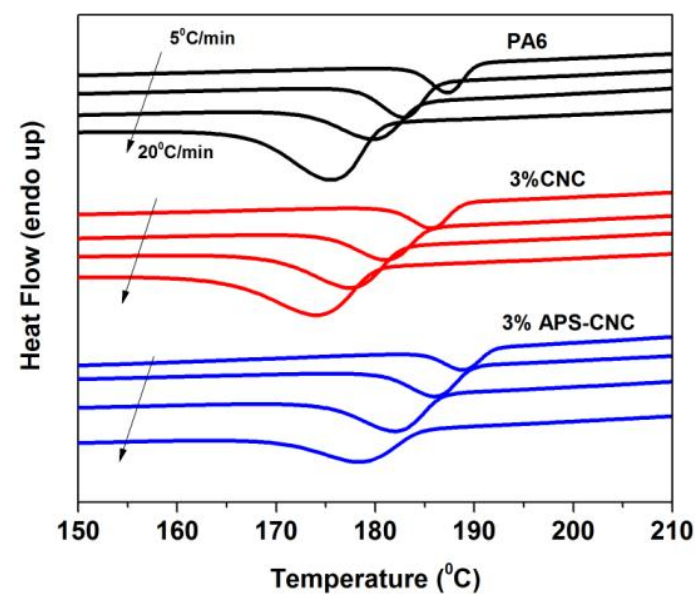

PA6 sample with that of $3 \% \mathrm{CNC}$ and $3 \%$ APS-CNC, it is evident in Table 4 that the peak crystallization temperature is slightly decreased in the case of $3 \% \mathrm{CNC}$ sample, while it shifts to higher temperatures for the APS modified CNC samples. This clearly shows that the surface modified CNCs cause the PA6 crystallization to occur at higher temperatures.

A number of theoretical approaches are used here to extract the crystallization kinetics parameters and to interpret the experimental results as follows: A constant cooling rate and similar thermal history applied to all the samples in the DSC furnace in all the experiments and the following equation (12) can be used in order to convert the crystallization temperature span to crystallization time for the purpose of explaining the observed crystallization kinetics of the samples:

$$
t=\left|\frac{T_{o}-T}{\varphi}\right|
$$


In equation (12), $T_{\mathrm{o}}$ is the onset of crystallization temperature, $T$ is the temperature at crystallization time of $t$ and $\varphi$ is the cooling rate. The typical variation of relative crystallinity versus temperature and time (obtained from the conversion just mentioned) for PA6 sample are shown in Figure 9. As can be seen in this figure, the variation of relative crystallinity versus both temperature and time follow a sigmoidal type behavior as expected. It is evident in Figure 9 that initially the crystallinity increases with a sharper curvature corresponding to the primary crystallization stage and, at higher degrees of relative crystallinity, the curve somewhat levels off with a very small curvature. Similar data trend was observed for 3\%CNC and 3\%APS-CNC samples (plots not shown).
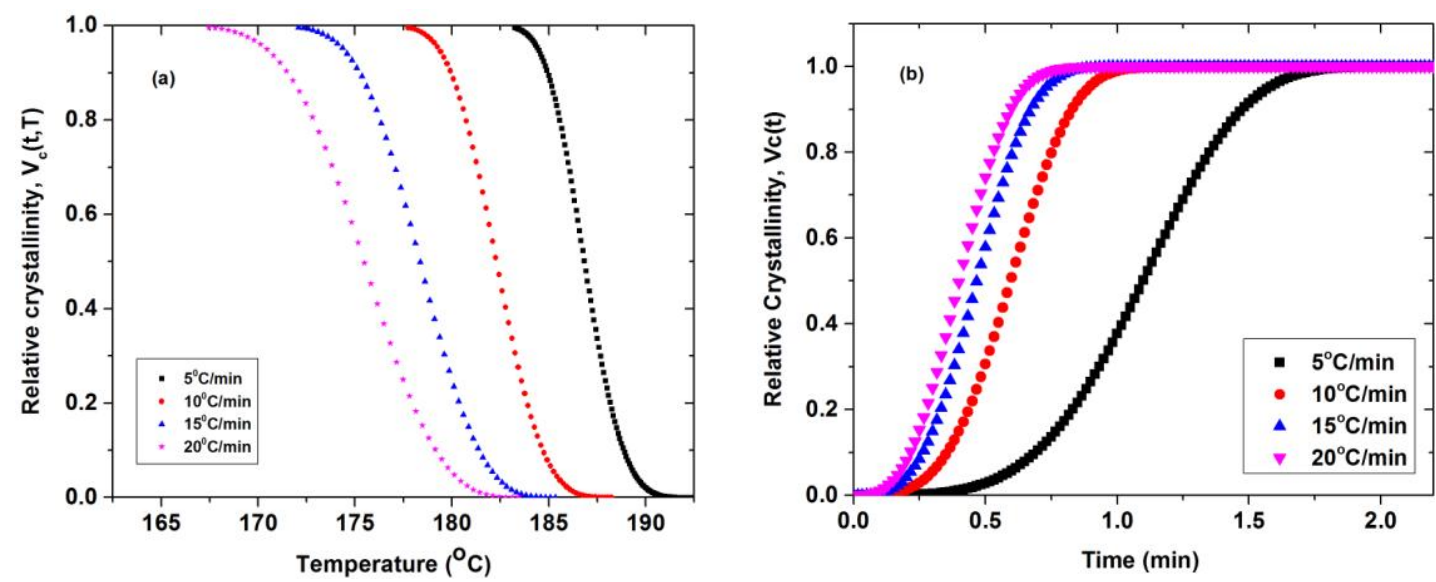

Figure 9: Variation of relative degree of crystallinity versus (a) temperature and (b) versus time for neat PA6 sample.

In the case of the PA6 sample, the curvature is relatively sharper which is indicative of a secondary crystallization. From the variation of relative crystallinity versus time it is evident that as the cooling rate increases, the time span of the crystallization decreases and the whole crystallization process takes place over a shorter period of time, indicating a faster crystallization rate (see Fig. 9).

\subsubsection{Avrami analysis for non-isothermal crystallization}

In order to adapt the Avrami model for analyzing non-isothermal crystallization process, a correction factor was used by Jeziorny [48] to modify the non-isothermal growth rate constant $\left(Z_{\mathrm{C}}\right)$ using the value derived directly from the Avrami equation $\left(Z_{t}\right)$ according to the following equations (13)-(15): 


$$
\begin{gathered}
1-X_{c}=\exp \left(-Z_{t} t^{n}\right) \\
\log \left[-\ln \left(1-X_{c}\right)\right]=n \log t+\log Z_{t} \\
\log Z_{C}=\frac{\log Z_{t}}{\varphi}
\end{gathered}
$$

The obtained modified Avrami plots of these three samples are shown in Figure 10 and the values of Avrami indices " $n$ ", the crystallization half times and the $Z_{\mathrm{t}}$ and $Z_{\mathrm{C}}$ are given in Table 4 .
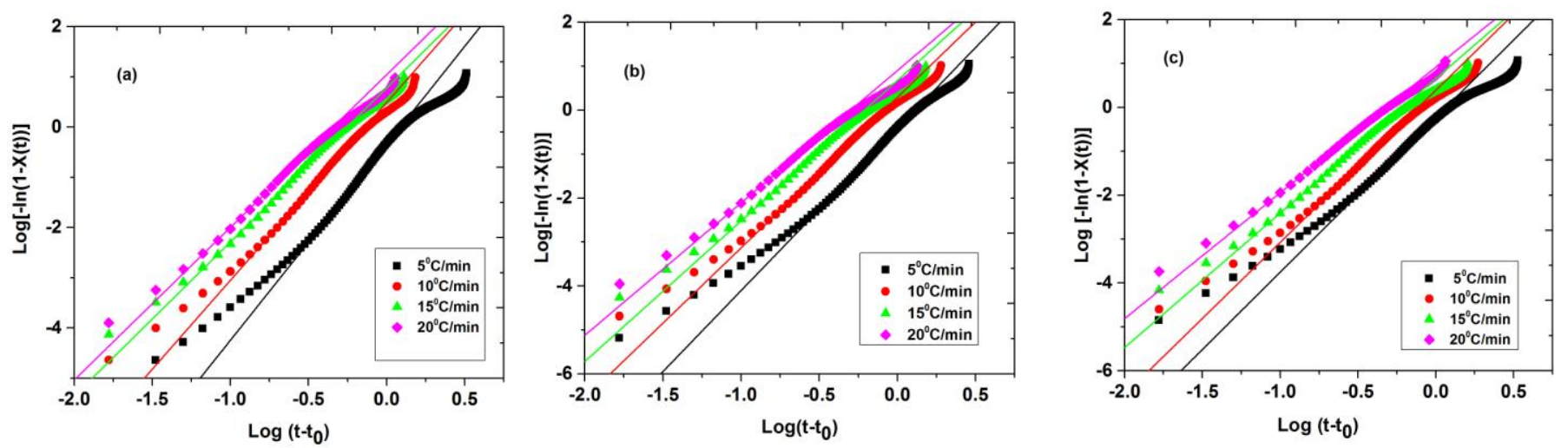

Figure 10: Avrami plots of non-isothermal crystallization of (a) PA6, (b)3\%CNC and (c)3\%APS$\mathrm{CNC}$

Clearly, Figure 10 and Table 4 show that the Avrami index for PA6 varies from 3.9 at lowest cooling rate (indicative of formation of relatively complex 3D shaped crystals) to 3 at highest cooling rate. This result shows that increasing the cooling rate from the melt results in development of less perfect PA6 crystals. In addition, both the nanocomposite samples with $3 \% \mathrm{CNC}$ and 3\%APS-CNC, show lower values of $n$ compared to that of the neat PA6, with the sample containing APS-CNC having lowest Avrami indices at all cooling rates. This shows that the surface modified $\mathrm{CNC}$ changed the crystal morphology to less perfect 3D crystals compared to that of the 3\%CNC sample. Another explanation for the reduction of $n$ as the cooling rate increases is the fact that higher cooling rates increase the crystallization rate, leading to possibility of spherulite impingement and truncation. This effect will be more important when the heterogeneous nuclei are dispersed non-uniformly (as in the case of the CNC particles) in addition to the increased surface area of the whiskers that are known to reduce the available volume for spherulite growth and therefore, a reduction of the obtained $n$ values. The obtained $t_{1 / 2}$ values show a 
decrease with increasing cooling rate for all samples which indicates enhanced crystallization rate (i.e., lower crystallization time) at higher cooling rates.

Table 4: Kinetic parameters for non-isothermal crystallization of PA6 and PA6 nanocomposites with 3\% of $\mathrm{CNC}$ and APS-CNC

\begin{tabular}{ccccccc}
\hline Samples & $\boldsymbol{\varphi}\left({ }^{\mathbf{0}} \mathbf{C} / \mathbf{m i n}\right)$ & $\mathbf{T}_{\mathbf{P}}\left({ }^{\mathbf{0}} \mathbf{C}\right)$ & $\mathbf{t}_{\mathbf{1} / \mathbf{2}}(\mathbf{m i n})$ & $\mathbf{n}$ & $\mathbf{Z}_{\mathbf{t}}$ & $\mathbf{Z}_{\mathbf{C}}$ \\
\hline \multirow{3}{*}{ PA6 } & 5 & 187.3 & 1.12 & 3.9 & 0.47 & 0.86 \\
& 10 & 182.9 & 0.62 & 3.6 & 3.11 & 1.11 \\
& 15 & 179.4 & 0.48 & 3.2 & 5.92 & 1.12 \\
& 20 & 176.8 & 0.42 & 3.0 & 11.65 & 1.13 \\
\hline \multirow{3}{*}{$\% \mathbf{C N C}$} & 5 & 185.6 & 1.18 & 3.7 & 0.38 & 0.82 \\
& 10 & 181.1 & 0.75 & 3.4 & 1.95 & 1.06 \\
& 15 & 177.6 & 0.58 & 3.2 & 4.52 & 1.10 \\
3\%APS-CNC & 20 & 174.4 & 0.48 & 3.0 & 7.94 & 1.11 \\
& 5 & 188.9 & 1.05 & 3.5 & 0.61 & 0.90 \\
& 10 & 186.1 & 0.71 & 3.4 & 2.63 & 1.10 \\
& 15 & 182.1 & 0.57 & 3.0 & 4.81 & 1.11 \\
& 20 & 178.4 & 0.46 & 2.8 & 8.34 & 1.11 \\
\hline
\end{tabular}

Comparing the 3\% CNC with the PA6 sample reveal that, at each cooling rate, the crystallization half-time is slightly increased. This shows that the pristine $\mathrm{CNC}$ whiskers prolong the crystallization process of PA6 chains. By contrast, the 3\%APS-CNC sample shows a relatively lower crystallization $t_{1 / 2}$ compared to that of the $3 \% \mathrm{CNC}$ sample, suggesting a partial compensation of the crystal growth delay by a plausible increased nucleation rate caused by enhanced surface activity.

Table 4 also shows the variation of $Z_{\mathrm{t}}$ and $Z_{\mathrm{C}}$ values which clearly show that increasing the cooling rate results in increasing crystallization rate constants which is consistent with the changes in crystallization half-time already discussed. By comparing the data obtained from the neat PA6 and the nanocomposite samples, it is evident that both 3\% CNC and APS-CNC samples show relatively lower overall crystallization rates compared to that of the neat PA6; while the values for APS modified CNC sample are found to be lower than that of the neat PA6 but higher than that of the 3\%CNC sample. The variation of Avrami rate constants show that the surface modified CNCs with better dispersion improve the crystallization rate compared to that of the nanocomposite samples containing the pristine $\mathrm{CNC}$ with fibrillar morphology. These findings combined with the fact that 3\%APS-CNC sample shifted the crystallization temperature to higher values (i.e., acceleration effect) suggest a complicated crystallization behavior that looks contradictory at first sight. However, these results can be explained as follows. The overall rate of crystallization is governed by the combination of rates of nucleation and crystal growth. The presence of solid 
surfaces of foreign particles in the polymer melt can act as heterogeneous nuclei while at the same time, these solid particles can decelerate the crystal growth due to the imposed restriction on macromolecular PA6 chain motion. The latter effect becomes significantly important and dominating if there are strong interactions between the host polymer and the surface of the particle. In the current PA6/CNC nanocomposite systems, the CNC whiskers show similar behavior where the strong hydroxyl rich surface of cellulose and amide groups of PA6 chains strongly interact through hydrogen bonding. In addition, the observed morphology of interconnected fibrillar network of whiskers result in severe restriction on molecular motion of PA6 that consequently results in lower overall crystallization rate, longer halt-time, and reduced Avrami exponent due to development of less perfect crystals. Similar effects have been observed with graphite oxide nano-sheets [49] and foliated graphite particles [33] in polyamide 6 matrix as reported in the literature. Further, in the case of APS modified CNC whiskers, because of the observed better dispersion of the particles throughout the matrix, the effect of particles on crystal growth hindrance is more prominent as already described. Here, we speculate that the higher interfacial area between the matrix and the whiskers and the interfacial bonds between the matrix and whiskers support the hypothesis of the $\mathrm{CNC}$ surface acting as heterogeneous secondary nuclei as observed in the isothermal crystallization and POM images already described. In fact, this same explanation is consistent with the observation of the onset and peak crystallization temperatures shifting towards higher temperatures in the case of 3\% APS-CNC sample.

\subsubsection{Ozawa kinetic model}

According to the theory reported by Ozawa $[50,51]$, non-isothermal crystallization process was assumed to be a combination of many infinitesimal isothermal crystallization processes over the duration of crystallization process where the cooling rate is constant. In this theory the relative crystallinity $X(\mathrm{~T})$ at each temperature $T$ can be written as equation (16) [52]:

$$
X(T)=1-\exp \left[\frac{-K(T)}{\varphi^{m}}\right]
$$

where $m$ is the Ozawa exponent and $K(\mathrm{~T})$ is the crystallization rate constant. This equation can be re-written in the linear form as:

$$
\log [-\ln (1-X(T))]=\log K(T)-m \log (\varphi)
$$


If this model is applicable to the non-isothermal crystallization of the current systems, a linear fit to the experimental data should accurately describe the data points and the graphical representation should generate a straight line. The results of the Ozawa analysis of our experimental data obtained at various crystallization temperatures (and corresponding $X(\mathrm{~T})$ at each cooling rate) are shown in Figure 11.
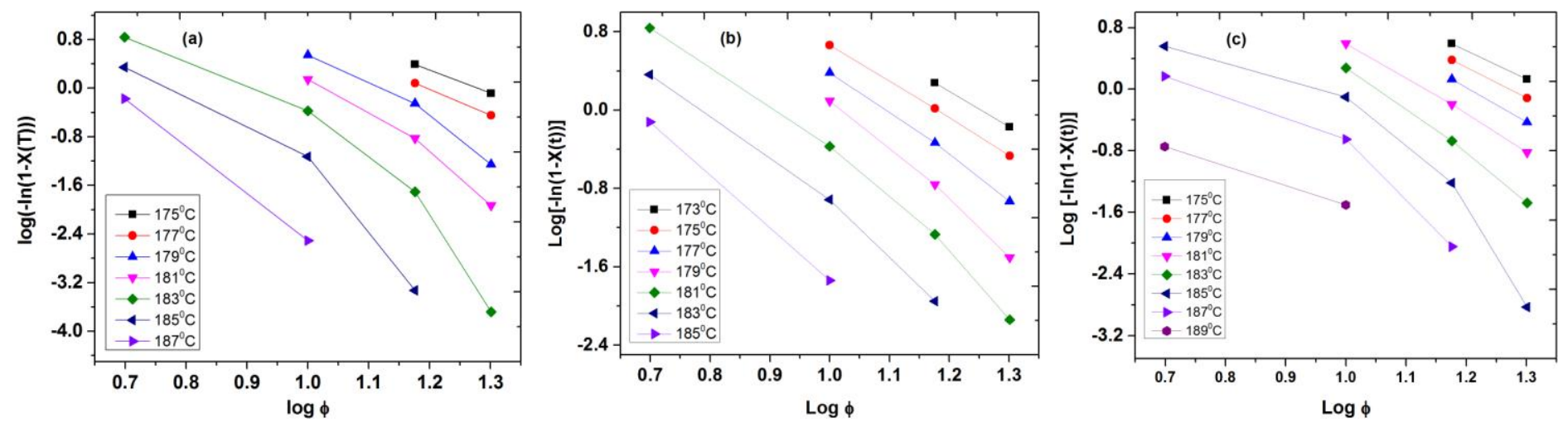

Figure 11: Ozawa plots of (a) PA6, (b) 3\%CNC and (c) 3\%APS-CNC

As can be seen from this figure, no obvious straight line could be fitted to the experimental data points, especially for PA6 and 3\%APS-CNC samples. Therefore, this model does not provide a satisfactory description of the obtained data. However, Figure 11 shows that the fit on the $3 \% \mathrm{CNC}$ data gives a relatively linear trend compared to that of the other samples. This finding might be related to the very slow crystallization rate of this particular sample and the specific network-like microstructure of the CNCs within the PA6 matrix, making the crystallization to occur near the diffusion limit regime. This observation is a typical behavior of systems where there is a significant molecular motion restriction imposed on the polymer chains [49]. Although the Ozawa model has been successfully fitted to data obtained on a number of polymeric and hybrid systems $[49,53]$, there are a number of cases reported in the literature where this model failed to fit nonisothermal crystallization data $[33,52]$. This may be ascribed to the fact that the non-isothermal crystallization process is a dynamic process where the crystallization rate constantly changes with both the time and temperature, making the assumptions of the quasi-isothermal nature of nonisothermal crystallization inadequate to describe this process especially at high heating rates like those used in this study. In addition, the Ozawa model ignores the secondary crystallization process [51] which is not true in the case of PA6 nanocomposites as it was earlier shown that the variation of relative crystallinity follows a sigmoidal dependence on the time and temperature with the upper curvature indicative of secondary crystallization process. Note that this last point may be less significant in the nanocomposite samples compared to the pure PA6 matrix due to steric 
factors and impingement of crystals with the solid surface of CNCs. In addition, in the case of 3\%APS-CNC sample, the experimental evidence suggests that the crystallization rate process is largely dependent on the extent of relative crystallinity which further complicates the process as will be discussed in the next section.

\subsubsection{Mo's approach towards non-isothermal crystallization}

As already discussed so far, both the Avrami and Ozawa's analysis methods did not provide accurate and clear description of the observed non-isothermal crystallization process. Therefore, as a further approach to address this limitation, Mo and coworkers [54] have proposed a model based on combination of Ozawa and Avrami models according to the following equations (18)-(19):

$$
\begin{gathered}
\ln K(T)-m \ln \varphi=\ln Z_{t}+n \ln t \\
\ln \varphi=\ln F(T)-\alpha \ln t
\end{gathered}
$$

where the $F(T)$ is defined as $\left[K(\mathrm{~T}) / Z_{\mathrm{t}}\right]^{1 / m}$ and the $\alpha$ is the ratio of Avrami exponent over Ozawa exponent $(\alpha=n / m)$. The physical meaning of the $F(T)$ parameter is the required cooling rate value in order to achieve a certain relative degree of crystallinity at unit crystallization time. If the left hand side of equation (19) is plotted against the $\ln t$ at a fixed degree of crystallinity, a straight line should be obtained with a slope of $\alpha$ and intercept of $\ln F(T)$. The significance of this model is the fact that the crystallization process can be discretized into various relative degrees of crystallinity and the kinetics can be studied at desired "stage" of crystallization.

The linear relationship between the $\ln \varphi$ and the $\ln t$ is demonstrated in Figure 12 where linear fits of equation (19) to experimental data obtained for PA6, 3\% CNC and 3\%APS-CNC samples at various relative degrees of crystallinity from $10 \%$ to $85 \%$ are plotted and the resulting fitting parameters of $F(T)$ and $\alpha$ are given in Table 5. The $F(T)$ parameter correlates with the crystallization rate at each relative degree of crystallization in such a way that the higher the $F(T)$ value, the lower the crystallization rate. This is because a high value of $F(T)$ means a higher cooling rate is required to reach the same degree of crystallinity which means that the crystallization is indeed occurring at lower rates [55]. Table 5 shows that the $F(T)$ values of $3 \% \mathrm{CNC}$ are higher compared to that of the PA6 and 3\%APS-CNC samples at all relative degrees of crystallinity considered, supporting the earlier findings that the $3 \% \mathrm{CNC}$ sample had relatively slower crystallization rate as already described. The most interesting part of this analysis, however, 
is the obtained $F(T)$ values for the 3\%APS-CNC sample which clearly shows that the $F(T)$ values ranged from 2.63 to 4.84 as a function of relative crystallinity ranging from 0.1 to 0.4 which corresponds to the early stages of crystallization or "nucleation phase" which is interestingly found to be lower than that of PA6 and 3\%CNC sample. This fact confirms our hypothesis that the APS modification actually enhances the nucleation activity of the CNC surfaces in the PA6 melt through interfacial bond formation with the polyamide 6 chains that facilitates development of the interphase layer that provides a path towards arrangement of macromolecular chains on the surface which in turn leads to enhanced heterogeneous nucleation. However, as the degree of crystallinity increases above $40 \%$, the $F(T)$ values increase rapidly to a level surpassing the $F(T)$ values of PA6 matrix.
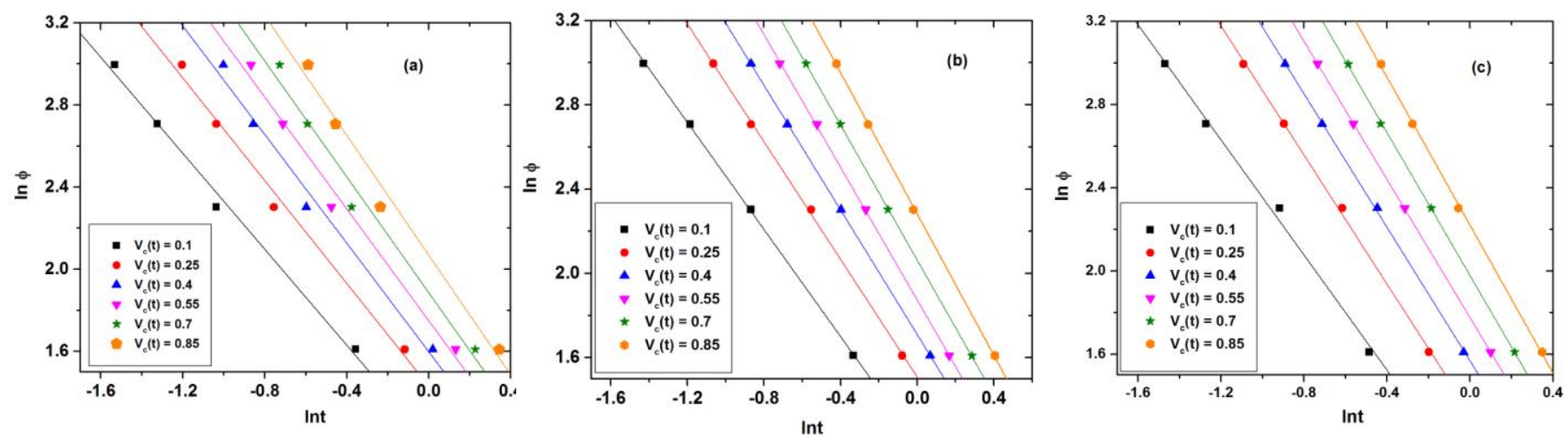

Figure 12: Ln $\varphi$ vs. Lnt from the Mo's equation for (a) PA6, (b) 3\%CNC and (c) 3\%APS-CNC nanocomposites

This last observation is consistent with the prior analysis showing the overall crystallization rate of this sample to be lower than that of neat PA6 (but still higher than 3\%CNC) at these later stages of crystallization due to the growth of the crystals becoming significantly retarded in the presence of well dispersed CNC whiskers and associated strong adhesion at the whisker/polymer interface that restricts the viscous motion of chain segments as already described. The variations in the slope of the fitted lines of equations (18) \& (19) to the experimental data show that addition of both CNC and APS-CNC nanoparticles increased the $\alpha$ value as shown in the Table 5. This means that the presence of $\mathrm{CNC}$ whiskers affects the Avrami exponent more significantly compared to the Ozawa exponent. The increase in the values of $\alpha$ with the relative degree of crystallinity show an increasing trend which can be attributed to the fact that at higher degrees of crystallinity, the variation of the crystallinity follows a relatively sharper dependence on the cooling rate which clearly is due to the restricted crystal growth at the later stages of the spherulitic development. 
Table 5: Results of Mo's analysis kinetic parameters at various relative degrees of crystallinity

\begin{tabular}{ccccccc}
\hline Relative degree of & \multicolumn{2}{c}{ PA6 } & \multicolumn{2}{c}{ 3\% CNC } & \multicolumn{2}{c}{ 3\%APS-CNC } \\
\cline { 2 - 7 } crystallinity & $\boldsymbol{\alpha}$ & $\mathbf{F}(\mathbf{T})$ & $\boldsymbol{\alpha}$ & $\mathbf{F}(\mathbf{T})$ & $\boldsymbol{\alpha}$ & $\mathbf{F}(\mathbf{T})$ \\
\hline $\mathbf{0 . 1}$ & 1.16 & 3.20 & 1.27 & 3.29 & 1.38 & 2.63 \\
$\mathbf{0 . 2 5}$ & 1.25 & 4.18 & 1.40 & 4.52 & 1.55 & 3.72 \\
$\mathbf{0 . 4}$ & 1.32 & 4.94 & 1.48 & 5.53 & 1.60 & 4.84 \\
$\mathbf{0 . 5 5}$ & 1.36 & 5.74 & 1.58 & 6.51 & 1.66 & 5.91 \\
$\mathbf{0 . 7}$ & 1.41 & 6.46 & 1.60 & 7.87 & 1.72 & 7.23 \\
$\mathbf{0 . 8 5}$ & 1.45 & 7.88 & 1.69 & 9.78 & 1.77 & 9.21 \\
\hline
\end{tabular}

\subsection{Non-isothermal crystallization activation energy}

The activation energy of the crystallization process is an important kinetic parameter to understanding and interpreting the interrelation of microstructure and crystallization process. There are a number of methods used to obtain the activation energy of typical non-isothermal crystallization process. One of the most commonly used methods is the Kissinger equation [56]. According to this method, a single value of activation energy can be obtained from the slope of the plot of $\ln \left(\varphi / T_{\mathrm{P}}{ }^{2}\right)$ versus $1 / T_{\mathrm{P}}\left(T_{\mathrm{p}}\right.$ is the peak crystallization temperature). However, the Kissinger and other Kissinger-type equations have some important disadvantages especially with respect to the nanocomposites systems of this current study, the most important of which, is that it provides a single activation energy value for the whole crystallization process. As already demonstrated, the non-isothermal crystallization process is a complicated sequence of nucleation and growth, and the crystallization rate is a function of relative degree of crystallinity. As an alternative approach to this method, a number of isoconversional methods have been developed to evaluate the activation energy discretized over relative crystallinity degree [51]. The representative methods include the differential isoconversion method of Friedman [57] and the Vyazovkin's advanced integral isoconversion approach. In the current study, we adopted the Friedman's differential isoconversion approach that is based on the numerical differentiation of conversion over crystallization time and on using the following equation:

$$
\ln \left(\frac{d X}{d t}\right)_{X, i}=\text { constant }-\frac{\Delta E_{X}}{R T_{X, i}}
$$

In this equation (20), $\mathrm{d} X / \mathrm{d} t$ is the instantaneous rate of crystallization at a given conversion. In order to apply this method, the values of relative degrees of crystallinity are numerically 
differentiated with respect to time to obtain the $\mathrm{d} X / \mathrm{d} t$ values. This process is carried out on a series of conversion points and the left hand side of the equation is correlated with the temperature of crystallization at that specific conversion point so that the discretized activation energy can be obtained from the slope. A typical plot of equation (20) is shown in Figure 13(a) for neat PA6 sample. A similar type of plot is constructed for 3\% CNC and 3\%APS-CNC samples and the variation of the obtained activation energies versus relative degree of crystallization is plotted in Figure 13(b).
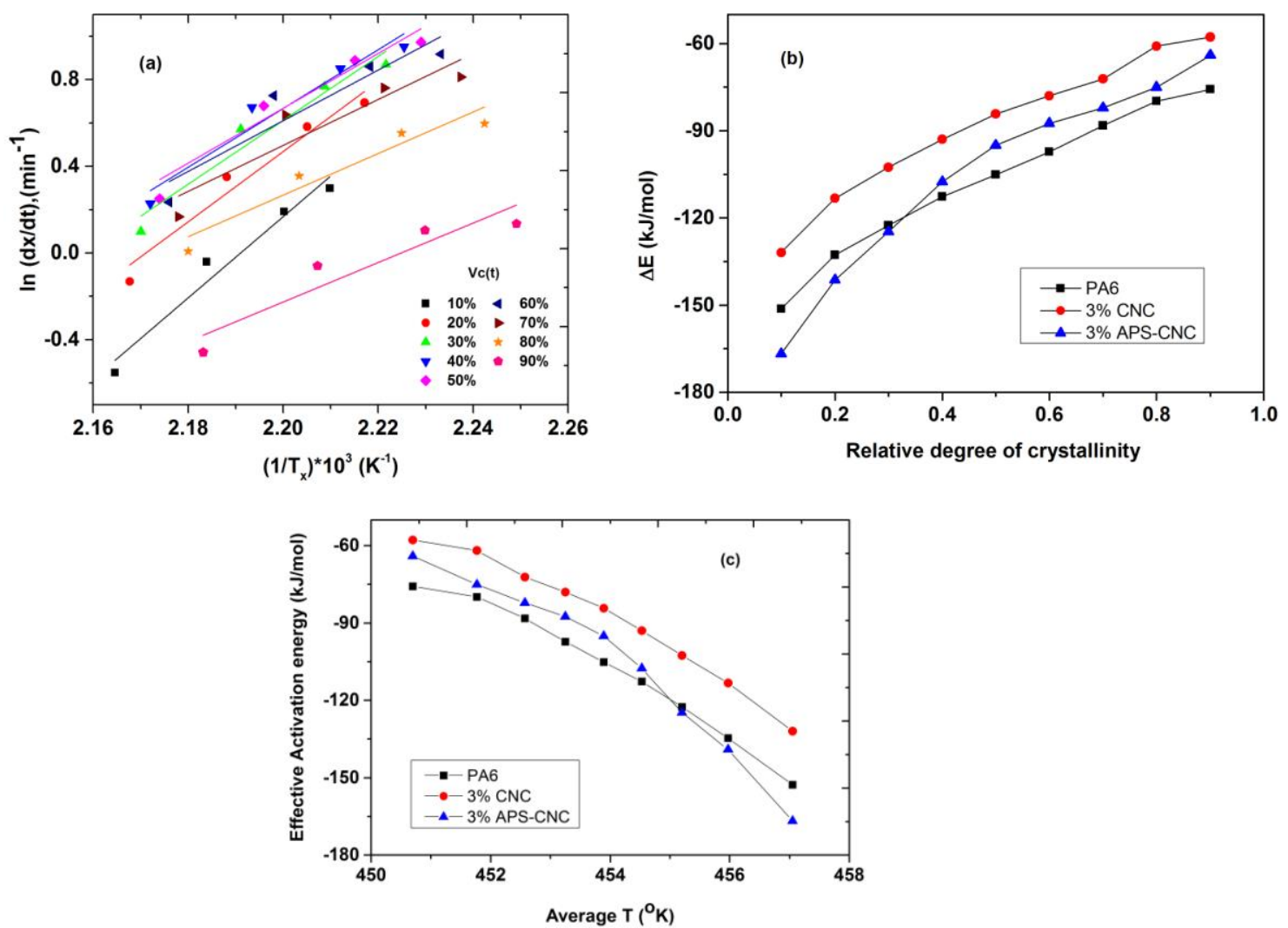

Figure 13: (a) Friedman plots of $\ln (\mathrm{d} X / \mathrm{d} t)$ vs. $1 / T_{X}$ for the PA6 at different relative degrees of crystallinity, (b) Dependence of the effective energy barrier on the relative degree of crystallinity and (c) dependence of the effective energy barrier on average temperature of PA6, 3\% CNC and $3 \%$ APS-CNC nanocomposites

As can be seen from Figure 13, the activation energy for all samples increases monotonically as the relative degree of crystallization is increased. This shows that as the fraction of crystalline material increases in the sample, it becomes harder for the macromolecular PA6 chains to further expand the growing spherulite, making this process relatively more energetically difficult. By comparing the data obtained for the nanocomposite samples with that of the PA6, it is clearly evident that the 3\%CNC sample shows higher activation energies at all stages of crystallization 
from $10 \%$ to $90 \%$. On the other hand, it is very interesting to note that the $3 \%$ APS-CNC sample showed a low initial activation energy from 10 to $40 \%$ of relative crystallinity. However, the obtained activation energy values surpass that of the PA6 sample for higher crystallization degrees above $40 \%$. This clearly shows that in the early stages of crystallization which corresponds to the nucleation phase, the crystal development is more kinetically favorable in the presence of surface modified CNC particles. In addition, as the crystallization fraction increases, this process becomes relatively more difficult as already described.

The schematic shown in Figure 14 is proposed [58] for development of the $\gamma$-type crystalline structure on the surface of APS-CNC particles as shown in this study. As previously mentioned, the $\alpha$-type crystals are formed through the hydrogen bonding of the amide linkages on chains in antiparallel arrangement in fully extended chain configuration therefore forming the H-bonded sheets of antiparallel chains. On other hand, the $\gamma$-type crystals are formed between the parallel sheets where the amide linkages are twisted $60^{\circ}$ out of the plane of the methyl groups. In our system, although the hydroxyl-rich surface of the CNC strongly interacts with PA6 chains, the arrangement of the antiparallel chains could be achieved as depicted in Figure 14; however, In the case of the APS-modified cellulose whiskers, the grafted chains of nylon 6 are chemically locked (tethered) on the surface which forces the nylon chains out of the ordered H-bonded sheets of the

(a)

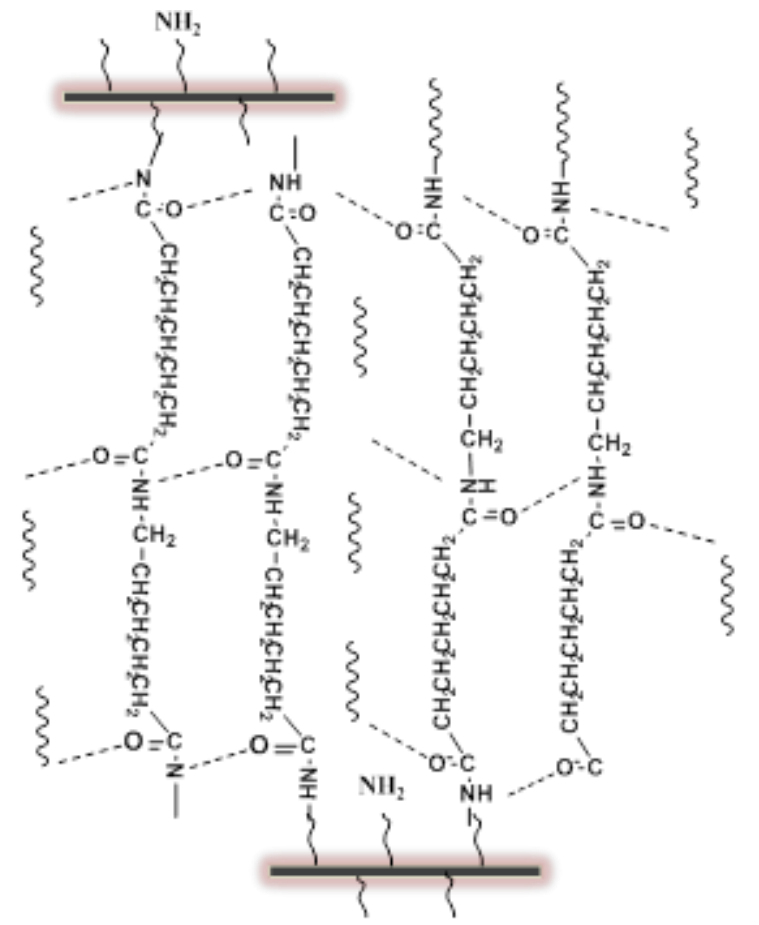

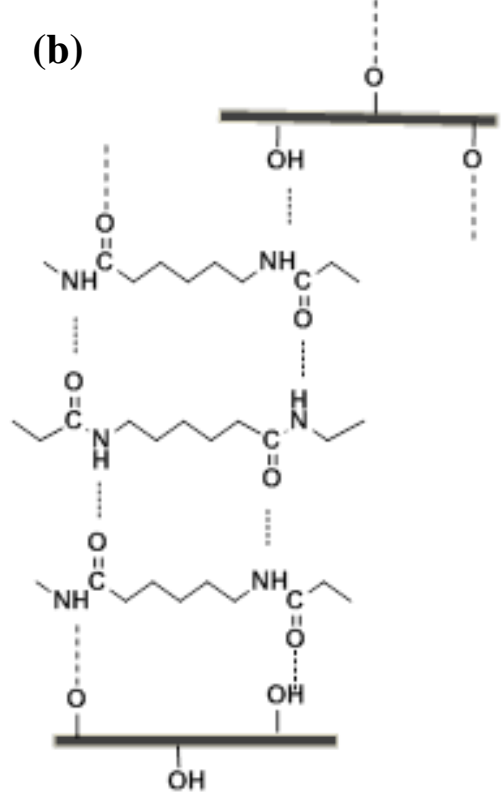

Figure 14: Schematic representation of (a) the $\gamma$-crystal structure formed at the interfacial area of the APS-modified CNC particles and (b) $\alpha$-crystal formation in absence of the grafted chains 
$\alpha$-structure. As a result, a greater number of $\gamma$-type crystals where less order is attained are formed. In addition, the SEM images showed a significantly improved dispersion of CNC within the matrix therefore, much higher surface area on the CNC is available for this effect which consequently, results in enhanced $\gamma$-nucleation effect.

\section{Conclusions}

This study showed that the crystallization kinetics and morphology of the nanocomposites of PA6 with cellulose nanocrystals (CNCs) and APS-modified CNCs prepared by in-situ ring opening polymerization and subsequent melt extrusion can be interpreted by analytic crystallization model equations with improved insights on the effects of the micro-structure development and surface properties of the nanocellulose needed to control the crystallization process of polyamide 6 . The SEM images showed a network-like fibrillar microstructure development in the case of non-modified PA6/CNC while in the case of PA6/APS-CNC system, a finer and more homogenous distribution of cellulose whiskers at sub-micron level was observed. For analysis of the crystallization kinetics, the Avrami model was successful in describing the isothermal crystallization process. The addition of CNCs resulted in longer crystallization halftimes and reduction in Avrami rate constant, indicating a hindrance effect of the cellulose nanocrystals on the crystallization process. This effect correlated well with the network-like fibrillar structure of the $\mathrm{CNC}$ in the PA6 matrix which imposes significant restriction on molecular motion and crystal development of PA6 macromolecular chains. However, the APS-modified CNC particles enhanced the observed nucleation activity and increased the Avrami $(K)$ constant. The effect of nucleation activity of the surface modified CNCs was confirmed by the Hoffman theory and polarized optical micrographs. In the non-isothermal crystallization, it was observed that the APS-modified CNCs shifted the crystallization peak and its onset temperature towards higher temperatures while an opposite effect was observed for the non-modified CNCs. The effective heterogeneous nucleation activity of the APS-CNCs was theoretically demonstrated below the $40 \%$ relative crystallinity range showing an excellent agreement with the experimental observation. The analysis of non-isothermal activation energy revealed that during the nucleation phase, the activation energy of the APS-modified CNC nanocomposite was lower than that of the PA6 and non-modified CNC system while this value surpassed that of the neat PA6 in later stages 
of non-isothermal crystallization. This study demonstrated how surface modification of cellulose nanocrystals and the associated microstructure development can effectively be used to control the crystallization behavior of polyamide 6 through balancing the competition between PA6 macromolecular motion chain restriction and heterogeneous nucleation activity.

\section{Acknowledgements}

The authors are grateful to Todd M. Alam at Sandia National Laboratories for conducting the solid state NMR experiment. The financial support by the U.S. National Science Foundation Division of Civil, Mechanical and Manufacturing Innovation through CMMI-1161292 grant award is gratefully acknowledged.

\section{References:}

[1] P. Matthies, W.F. Seydl, High Performance Polymers: Their Origin and Development: Proceedings of the Symposium on the History of High Performance Polymers at the American Chemical Society Meeting held in New York, April 15-18, 1986, Springer Netherlands, Dordrecht, 1986, pp. 39-53.

[2] I. Page, Polyamides as engineering thermoplastic materials, iSmithers Rapra Publishing, 2000.

[3] K. Miyasaka, K. Ishikawa, J. Polym. Sci., Part A-2: Polym. Phys., 6 (1968) 1317-1329.

[4] K. Miyasaka, T. Isomoto, H. Koganeya, K. Uehara, K. Ishikawa, N. Ogata, Journal of Polymer Science: Polymer Physics Edition, 18 (1980) 1047-1052.

[5] N.S. Murthy, J. Polym. Sci., Part B: Polym. Phys., 44 (2006) 1763-1782.

[6] N.S. Murthy, H. Minor, R.A. Latif, J. Macromol. Sci., Part B: Phys., 26 (1987) 427-446.

[7] H. Arimoto, M. Ishibashi, M. Hirai, Y. Chatani, J. Polym. Sci. Part A: General Papers, 3 (1965) 317-326.

[8] X. Liu, Q. Wu, Eur. Polym. J., 38 (2002) 1383-1389.

[9] S.C. Tjong, S.P. Bao, J. Polym. Sci., Part B: Polym. Phys., 42 (2004) 2878-2891.

[10] P.V. Kodgire, A.R. Bhattacharyya, S. Bose, N. Gupta, A.R. Kulkarni, A. Misra, Chem. Phys. Lett., 432 (2006) 480-485.

[11] Liu, I.Y. Phang, L. Shen, S.Y. Chow, W.-D. Zhang, Macromolecules, 37 (2004) 7214-7222. 
[12] F. Zhang, X. Peng, W. Yan, Z. Peng, Y. Shen, J. Polym. Sci., Part B: Polym. Phys., 49 (2011) 1381-1388.

[13] F. Yang, Y. Ou, Z. Yu, J. Appl. Polym. Sci., 69 (1998) 355-361.

[14] T.-M. Wu, Y.-H. Lien, S.-F. Hsu, J. Appl. Polym. Sci., 94 (2004) 2196-2204.

[15] T.D. Fornes, D.R. Paul, Polymer, 44 (2003) 3945-3961.

[16] B. Guo, Q. Zou, Y. Lei, M. Du, M. Liu, D. Jia, Thermochim. Acta, 484 (2009) 48-56.

[17] J. Li, Z. Fang, L. Tong, A. Gu, F. Liu, Eur. Polym. J., 42 (2006) 3230-3235.

[18] J. Li, Z. Fang, Y. Zhu, L. Tong, A. Gu, F. Liu, J. Appl. Polym. Sci., 105 (2007) 3531-3542.

[19] R.J. Moon, A. Martini, J. Nairn, J. Simonsen, J. Youngblood, Chem. Soc. Rev., 40 (2011) 3941-3994.

[20] M.T. Postek, R.J. Moon, A.W. Rudie, M.A. Bilodeau, Technical Association of Pulp and Paper Industry, TAPPI, Peachtree Corners, GA, (2013).

[21] J.K. Pandey, C.S. Lee, S.-H. Ahn, J. Appl. Polym. Sci., 115 (2010) 2493-2501.

[22] J. Han, Y. Zhu, J. Hu, H. Luo, L.-Y. Yeung, W. Li, Q. Meng, G. Ye, S. Zhang, Y. Fan, J. Appl. Polym. Sci., 123 (2012) 749-762.

[23] G. Siqueira, C. Fraschini, J. Bras, A. Dufresne, R. Prud'homme, M.-P. Laborie, Eur. Polym. J., 47 (2011) 2216-2227.

[24] J. Lu, T. Wang, L.T. Drzal, Compos Part A-Appl S , 39 (2008) 738-746.

[25] A. Jalal Uddin, J. Araki, Y. Gotoh, Polym. Int., 60 (2011) 1230-1239.

[26] A. Kiziltas, B. Nazari, D.J. Gardner, D.W. Bousfield, Polym. Eng. Sci., 54 (2014) 739-746.

[27] S. Kashani Rahimi, J.U. Otaigbe, Polym. Eng. Sci., 56 (2016) 1045-1060.

[28] J.R. Capadona, O. Van Den Berg, L.A. Capadona, M. Schroeter, S.J. Rowan, D.J. Tyler, C. Weder, Nat Nano, 2 (2007) 765-769.

[29] R. Mateva, P. Petrov, S. Rousseva, R. Dimitrov, G. Zolova, Eur. Polym. J., 36 (2000) 813821.

[30] K. Udipi, R.S. Davé, R.L. Kruse, L.R. Stebbins, Polymer, 38 (1997) 927-938.

[31] T. Weeding, W. Veeman, H.A. Gaur, W. Huysmans, Macromolecules, 21 (1988) 2028-2032.

[32] L. Liu, Z. Qi, X. Zhu, J. Appl. Polym. Sci., 71 (1999) 1133-1138.

[33] W. Weng, G. Chen, D. Wu, Polymer, 44 (2003) 8119-8132. 
[34] A.T. Lorenzo, M.L. Arnal, J. Albuerne, A.J. Müller, Polym. Test., 26 (2007) 222-231.

[35] M. Fanfoni, M. Tomellini, Il Nuovo Cimento D, 20 (1998) 1171-1182.

[36] J. Málek, Thermochim. Acta, 267 (1995) 61-73.

[37] W.Y. Zhou, B. Duan, M. Wang, W.L. Cheung, Advances in diverse industrial applications of nanocomposites. ISBN, (2011) 978-953.

[38] E.-C. Chen, T.-M. Wu, J. Polym. Sci., Part B: Polym. Phys., 46 (2008) 158-169.

[39] J.D. Hoffman, G.T. Davis, J.I. Lauritzen, Treatise on Solid State Chemistry: Volume 3 Crystalline and Noncrystalline Solids, Springer US, Boston, MA, 1976, pp. 497-614.

[40] L.H. Sperling, Introduction to physical polymer science, John Wiley \& Sons, 2005.

[41] J.D. Hoffman, J.J. Weeks, J Res Natl Bur Stand A, 66 (1962) 13-28.

[42] J.-C. Ho, K.-H. Wei, Macromolecules, 33 (2000) 5181-5186.

[43] N. Barhoumi, A. Maazouz, M. Jaziri, R. Abdelhedi, Express Polym Lett, 7 (2013) 76-87.

[44] E. Lizundia, J. Vilas, L. León, Carbohydr. Polym., 123 (2015) 256-265.

[45] M.-J. Chu, T.-M. Wu, Experimental Analysis of Nano and Engineering Materials and Structures, Springer, 2007, pp. 827-828.

[46] Y. Katoh, M. Okamoto, Polymer, 50 (2009) 4718-4726.

[47] D. Wu, C. Zhou, X. Fan, D. Mao, Z. Bian, J. Appl. Polym. Sci., 99 (2006) 3257-3265.

[48] A. Jeziorny, Polymer, 19 (1978) 1142-1144.

[49] Y. Liu, G. Yang, Thermochim. Acta, 500 (2010) 13-20.

[50] T. Ozawa, Polymer, 12 (1971) 150-158.

[51] G.Z. Papageorgiou, D.S. Achilias, D.N. Bikiaris, G.P. Karayannidis, Thermochim. Acta, 427 (2005) 117-128.

[52] M. Kuo, J. Huang, M. Chen, Mater. Chem. Phys., 99 (2006) 258-268.

[53] P. Supaphol, N. Dangseeyun, P. Srimoaon, M. Nithitanakul, Thermochim. Acta, 406 (2003) 207-220.

[54] T. Liu, Z. Mo, H. Zhang, J. Appl. Polym. Sci., 67 (1998) 815-821.

[55] H. Huang, L. Gu, Y. Ozaki, Polymer, 47 (2006) 3935-3945.

[56] H.E. Kissinger, J. Res. Nat. Bur. Stand. 57 (1956) 217-221. 
[57] H.L. Friedman, in: J. Polym. Sci. Part C: Polymer Symposia, Wiley Online Library, 1964, pp. 183-195.

[58] J. Li, Z. Fang, L.Tong, A. Gu, F. Liu, J. Polym. Sci., Part B: Polym. Phys., 44 (2006) 14991512 

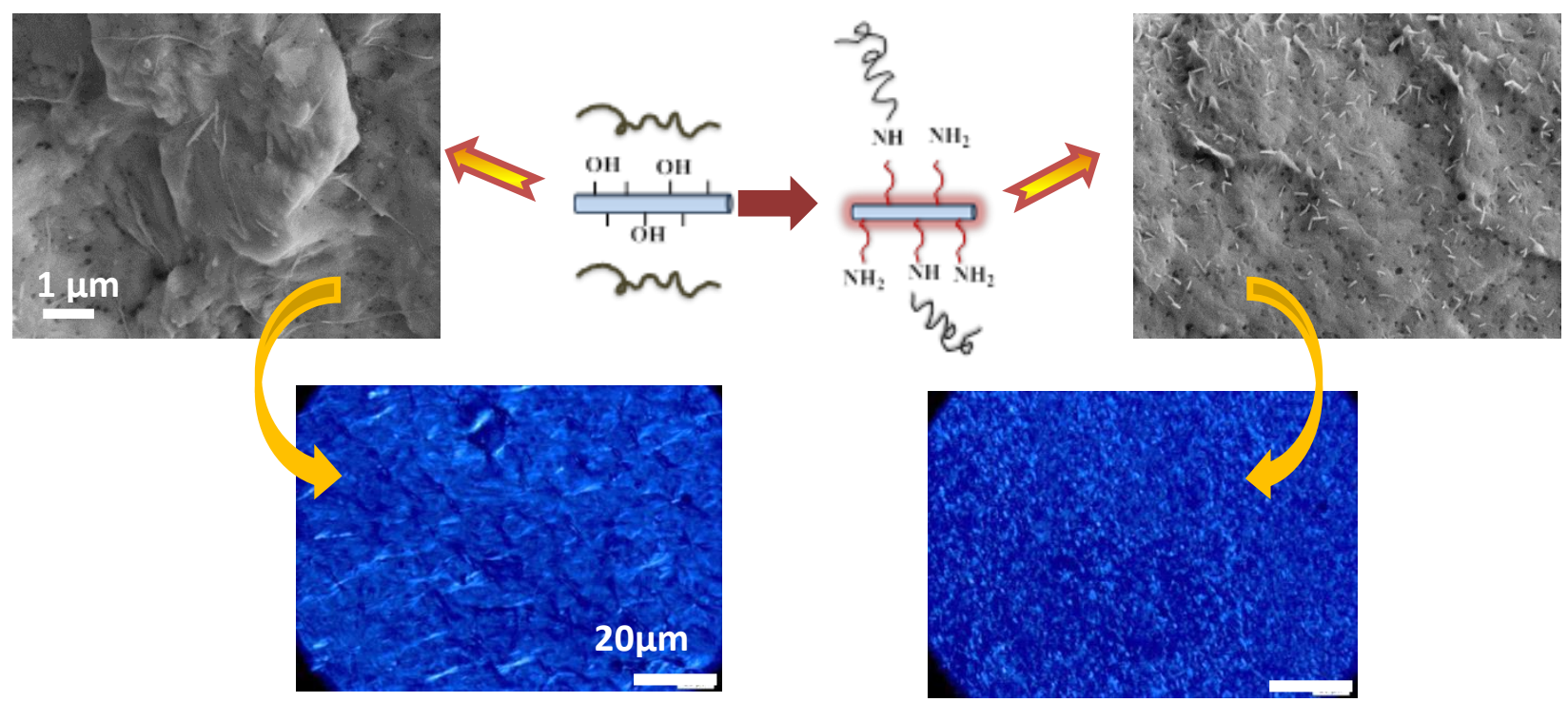